Pure and Applied Mathematics Quarterly

Volume 5, Number 3

(Special Issue: In honor of

Friedrich Hirzebruch, Part 2 of 2$)$

$873-914,2009$

\title{
Topological Rigidity for Non-aspherical Manifolds
}

\author{
M. Kreck and W. Lück
}

\begin{abstract}
The Borel Conjecture predicts that closed aspherical manifolds are topological rigid. We want to investigate when a non-aspherical oriented connected closed manifold $M$ is topological rigid in the following sense. If $f: N \rightarrow M$ is an orientation preserving homotopy equivalence with a closed oriented manifold as target, then there is an orientation preserving homeomorphism $h: N \rightarrow M$ such that $h$ and $f$ induce up to conjugation the same maps on the fundamental groups. We call such manifolds Borel manifolds. We give partial answers to this questions for $S^{k} \times S^{d}$, for sphere bundles over aspherical closed manifolds of dimension $\leq 3$ and for 3-manifolds with torsionfree fundamental groups. We show that this rigidity is inherited under connected sums in dimensions $\geq 5$. We also classify manifolds of dimension 5 or 6 whose fundamental group is the one of a surface and whose second homotopy group is trivial.
\end{abstract}

Keywords: Topological rigidity, Borel Conjecture, classification of lowdimensional topological manifolds.

Mathematics Subject Classification 2000: 57N99, 57R67.

\section{Introduction and Statement of Results}

In this paper we study the question which non-aspherical oriented closed connected topological manifolds are topological rigid. Recall that the Borel Conjecture predicts that every aspherical closed topological manifold is topological rigid in the sense that every homotopy equivalence of such manifolds is homotopic to a

Received September 12, 2005. 
homeomorphism. We focus on the following two problems which we will describe next.

We say that two maps $f, g: X \rightarrow Y$ of path-connected spaces induce the same map on the fundamental groups up to conjugation if for one (and hence all base points) $x \in X$ there exists a path $w$ from $f(x)$ to $g(x)$ such that for the group isomorphism $t_{w}: \pi_{1}(Y, f(x)) \rightarrow \pi_{1}(Y, g(x))$ which sends the class of a loop $v$ to the class of the loop $w^{-} * v * w$ we get $\pi_{1}(g, x)=t_{w} \circ \pi_{1}(f, x)$. Homotopic maps induce the same map on the fundamental groups up to conjugation.

Convention 0.1. Manifold will always mean connected oriented closed topological manifold unless stated explicitly differently.

Definition 0.2 (Borel-manifold). A manifold $M$ is called a Borel manifold if for any orientation preserving homotopy equivalence $f: N \rightarrow M$ of manifolds there exists an orientation preserving homeomorphism $h: N \rightarrow M$ such that $f$ and $h$ induce the same map on the fundamental groups up to conjugation. It is called a strong Borel manifold if every orientation preserving homotopy equivalence $f: N \rightarrow M$ of manifolds is homotopic to a homeomorphism $h: N \rightarrow M$.

Remark 0.3 (Relation to the Borel Conjecture). If $M$ is aspherical, two homotopy equivalences $f, g: N \rightarrow M$ are homotopic if and only if they induce the same map on the fundamental groups up to conjugation. Hence an aspherical manifold $M$ is a Borel manifold if and only if every homotopy equivalence $f: N \rightarrow M$ of manifolds is homotopic to a homeomorphism. This is the precise statement of the Borel Conjecture for aspherical manifolds. Hence the Borel Conjecture can be rephrased as the statement that every aspherical manifold $M$ is a Borel manifold, or equivalently, is a strong Borel manifold. More information on the Borel Conjecture can be found for instance in [9], [10], [11], [12], [14], [15], [16], [24], [26], [27].

Remark 0.4 (Relation to the Poincaré Conjecture). The statement that $S^{n}$ is a strong Borel manifold is equivalent to the Poincaré Conjecture that every manifold which is homotopy equivalent to a sphere $S^{n}$ is homeomorphic to $S^{n}$. This follows from the fact that there are exactly two homotopy classes of self-homotopy equivalences $S^{n} \rightarrow S^{n}$ which both have homeomorphisms as representatives. In particular $S^{n}$ is a Borel manifold if and only if it is a strong Borel manifold.

Problem 0.5 (Classification of Borel manifolds). Which manifolds are Borel manifolds? 
In the light of both the Borel Conjecture and the Poincaré Conjecture, it is natural to consider the class of manifolds $M$, whose universal covering $\widetilde{M}$ is homotopy-equivalent to a wedge of $k$-spheres $S^{k}$ for some $2 \leq k \leq \infty$. We call such a manifold a generalized topological space form. If $k \neq \infty$, this condition is equivalent to saying that the reduced integral homology vanishes except in dimension $k$ and it is a direct sum of copies of $\mathbb{Z}$ in dimension $k$. If $k=\infty$, then this condition is equivalent to saying that $M$ is an aspherical manifold. A simply-connected generalized topological space form is the same as a homotopy sphere. More generally, a generalized topological space form with finite fundamental group, is the same as a spherical topological space form. If $G$ acts freely and cocompactly and properly discontinuously on $S^{k} \times \mathbb{R}^{m-k}$, then $M=S^{k} \times \mathbb{R}^{m-k} / G$ is a generalized topological space form. If $M$ and $N$ are $m$ dimensional aspherical manifolds, then $M \# N$ is a generalized topological space form. If $M$ is aspherical, then for each $k$ the manifold $M \times S^{k}$ is a generalized space form, or more generally, all $S^{k}$-bundles over $M$ with $k>1$ are generalized space forms.

Most results in this paper concern generalized space forms $M$. One can try to attack the question whether $M$ is Borel by computing its structure set $S^{t o p}(M)$. It consists of equivalence classes of orientation preserving homotopy equivalences $N \rightarrow M$ with a manifold $N$ as source, where two such homotopy equivalences $f_{0}: N_{0} \rightarrow M$ and $f_{1}: N_{1} \rightarrow M$ are equivalent if there exists a homeomorphism $g: N_{0} \rightarrow N_{1}$ with $f_{1} \circ g \simeq f_{0}$. The group ho-aut ${ }_{\pi}(M)$ of homotopy classes of self equivalences inducing the identity on $\pi_{1}$ up to conjugation acts on this set by composition. A manifold $M$ is strongly Borel if and only if $S^{\text {top }}(M)$ consists of one element. A manifold $M$ is Borel if and only if ho-aut ${ }_{\pi}(M)$ acts transitively on $S^{t o p}(M)$. In general it is very hard to compute the structure set. But if the Farrell-Jones Conjecture for $G=\pi_{1}(M)$ holds, then one often can do this. More precisely we mean the Farrell-Jones Conjecture for $K$ - and $L$-theory for the group $G$. In all relevant cases $G$ will be torsionfree. Hence this phrase will mean that $\operatorname{Wh}(G)$ and $\widetilde{K}_{n}(\mathbb{Z} G)$ vanish for $n \leq 0$ and that the assembly map $H_{n}(B G ; \mathbf{L}) \rightarrow L_{n}(\mathbb{Z} G)$ is bijective for all $n \in \mathbb{Z}$, where $\mathbf{L}$ is the (non-connective periodic) $L$-theory spectrum and $L_{n}(\mathbb{Z} G)$ is the $n$-th quadratic $L$-group of $\mathbb{Z} G$. (We can ignore the decoration by the Rothenberg sequences and the assumption that $\mathrm{Wh}(G)$ and $\widetilde{K}_{n}(\mathbb{Z} G)$ vanish for $n \leq 0$.) More information about the FarrellJones Conjecture can be found for instance in [13], [24] and [27]. 
For example the Farrell-Jones Conjecture holds for $\mathbb{Z}$ and the fundamental group of surfaces of genus $\geq 1$. Combining this with the construction of certain self-homotopy equivalences, we obtain the following result concerning generalized topological space forms.

Theorem 0.6 (Sphere bundles over surfaces). Let $K$ be $S^{1}$ or a 2-dimensional manifold different from $S^{2}$. Let $S^{d} \rightarrow E \rightarrow K$ be a fiber bundle over $K$ such that $E$ is orientable and $d \geq 3$.

Then $E$ is a Borel manifold. It is a strong Borel manifold if and only if $K=S^{1}$.

This gives examples of Borel manifolds in all dimensions $>3$, which are neither homotopy spheres nor aspherical.

In dimension 3 the existence of Borel manifolds is related to the Poincaré Conjecture and to Thurston's Geometrization Conjecture. Results of Waldhausen and Turaev imply:

Theorem 0.7 (Dimension 3). Suppose that Thurston's Geometrization Conjecture for irreducible 3-manifolds with infinite fundamental group and the 3dimensional Poincaré Conjecture are true. Then every 3-manifold with torsionfree fundamental group is a strong Borel manifold.

Using the Kurosh theorem and the prime decomposition of 3-manifolds one can even show that if the assumptions of this theorem are fulfilled then the fundamental group determines the homeomorphism type, a close analogy between surfaces and 3-manifolds (although the latter case is of course much more complicated).

Recently Perelman has announced a proof of Thurston's Geometrization Conjecture but details are still checked by the experts.

Given the analogy between the classification of surfaces and 3-manifolds with torsionfree fundamental group, it is natural to study in analogy to sphere bundles over surfaces sphere bundles over 3-manifolds. Our result here is:

Theorem 0.8 (Sphere bundles over 3-manifolds). Let $K$ be an aspherical 3dimensional manifold. Suppose that the Farrell-Jones Conjecture for $K$ - and $L$-theory holds for $\pi_{1}(K)$. Let $S^{d} \rightarrow E \rightarrow K$ be a fiber bundle over $K$ with orientable $E$ such that $d \geq 4$ or such that $d=2,3$ and there is a map $i: K \rightarrow E$ with $p \circ i \simeq \operatorname{id}_{K}$. Then 
(a) $E$ is strongly Borel if and only if $H_{1}(K ; \mathbb{Z} / 2)=0$;

(b) If $d=3 \bmod 4$ and $d \geq 7$, then $K \times S^{d}$ is Borel;

(c) If $d=0 \bmod 4$ and $d \geq 8$ and $H_{1}(K ; \mathbb{Z} / 2) \neq 0$, then $K \times S^{d}$ is not Borel.

The following result shows that if the fundamental groups of two $d$-dimensional Borel manifolds $M$ and $N$ contain no 2-torsion, then the connected sum $M \# N$ is a Borel manifold. Here we assume that $d>4$.

Theorem 0.9 (Connected sums). Let $M$ and $N$ be manifolds of the same dimension $n \geq 5$ such that neither $\pi_{1}(M)$ nor $\pi_{1}(N)$ contains elements of order 2 or that $n=0,3 \bmod 4$. If both $M$ and $N$ are (strongly) Borel, then the same is true for their connected sum $M \# N$.

Remark 0.10. If $M$ and $N$ are aspherical Borel manifolds of dimension $\neq 4$ then $M \# N$ is a generalized topological space form, which is Borel.

Combining the previous results, we have found infinitely many non-aspherical and non-simply connected Borel manifolds in each dimension $\neq 4$. The proof is in all cases based on a determination of the structure set and by providing enough self equivalences following the scheme described above.

The main reason why these proofs do not work at present in dimension 4 is that for the fundamental groups under consideration it is not known whether they are good in the sense of Freedman. For this reason one has to look at other classes of 4-manifolds where also the determination of the structure set is known but it is not clear how to construct enough self equivalences to guarantee a transitive action. However, if $\pi_{1}(M)$ is cyclic and $M$ is a spin manifold, one can use other methods to show that the homotopy type determines the homeomorphism type (respecting the identification of fundamental groups). Previously known Borel 4-manifolds are flat 4-manifolds, where the Borel Conjecture was proven.

Theorem 0.11 (Dimension 4). $\quad$ (a) Let $M$ be a 4-manifold with Spin structure such that its fundamental group is finite cyclic. Then $M$ is Borel.

If $M$ is simply connected and Borel, then it has a Spin structure.

(b) Let $N$ be a flat smooth Riemannian 4-manifold or be $S^{1} \times S^{3}$. Then $N$ is strongly Borel. If $M$ is a simply connected 4-manifold with Spin-structure, then $M \# N$ is Borel. 
By Theorem 0.11(b) we have provided infinitely many non-aspherical and nonsimply connected Borel manifolds in dimension 4. Except for $S^{1} \times S^{3}$ and the flat manifolds, these manifolds are not generalized topological space forms.

We have seen that under some mild restrictions the connected sum of two Borel manifolds is a Borel manifold. It is natural to ask the corresponding question for the cartesian product of Borel manifolds. If $M$ and $N$ are aspherical, then $M \times N$ is aspherical and so Borel, if the Borel Conjecture holds. But if the manifolds are not aspherical Borel manifolds, the picture becomes complicated. An interesting test case is provided by the product of two spheres, where we give a complete answer in terms of the unstable Arf invariant.

Let $\Omega_{k, k+d}^{\mathrm{fr}}$ be the bordism set of smooth $k$-dimensional manifolds $M$ with an embedding $i: M \rightarrow \mathbb{R}^{k+d}$ together with an (unstable) framing of the normal bundle $\nu(i)$. If $d>k$, this is the same as the bordism group $\Omega_{k}^{\mathrm{fr}}$ of stably framed smooth $k$-dimensional manifolds since any $k$-dimensional smooth manifold $M$ admits an embedding into $\mathbb{R}^{k+d}$ and a stable framing on $\nu\left(M, \mathbb{R}^{k+d}\right)$ is the same as an unstable framing for $d>k$. The Arf invariant $\operatorname{Arf}(M) \in \mathbb{Z} / 2$ of a stably framed manifold $M$ whose dimension $k$ satisfies $k=2 \bmod 4$ is the Arf invariant of the surgery problem associated to any degree one map $M \rightarrow S^{\operatorname{dim}(M)}$ with the obvious bundle data coming from the stable framing. It induces a homomorphism of abelian groups for $k=2 \bmod 4$

$$
\operatorname{Arf}_{k}: \Omega_{k}^{\mathrm{fr}} \rightarrow \mathbb{Z} / 2 .
$$

If $g: S^{k} \times S^{d} \rightarrow S^{k}$ is a map for $d=2 \bmod 4$, define its $\operatorname{Arf}$ invariant $\operatorname{Arf}(g) \in$ $\mathbb{Z} / 2$ to be the Arf invariant of the stably framed manifold $\bar{g}^{-1}(\{\bullet\})$ for any map $\bar{g}: S^{k} \times S^{d} \rightarrow S^{k}$ which is homotopic to $g$ and transverse to $\{\bullet\} \subseteq S^{k}$. Here the stable framing of $\bar{g}^{-1}(\{\bullet\})$ is given by the standard stable framing of the normal bundle of $S^{k} \times S^{d}$ and the trivialization of the normal bundle of $\bar{g}^{-1}(\{\bullet\}) \subseteq S^{k} \times S^{d}$ coming from transversality.

Theorem 0.13 (Products of two spheres). Consider $k, d \in \mathbb{Z}$ with $k, d \geq 1$. Then:

(a) Suppose that $k+d \neq 3$. Then $S^{k} \times S^{d}$ is a strong Borel manifold if and only if both $k$ and $d$ are odd;

(b) For $d \neq 2$ the manifolds $S^{1} \times S^{d}$ is strongly Borel, and $S^{1} \times S^{2}$ is strongly Borel if and only if the 3-dimensional Poincaré Conjecture is true; 
(c) The manifold $S^{2} \times S^{2}$ is Borel but not strongly Borel;

(d) Suppose $k, d>1$ and $k+d \geq 4$. Then the manifold $S^{k} \times S^{d}$ is Borel if and only if the following conditions are satisfied:

(i) Neither $k$ nor $d$ is divisible by 4 ;

(ii) If $k=2 \bmod 4$, then there is a map $g_{k}: S^{k} \times S^{d} \rightarrow S^{k}$ such that its Arf invariant $\operatorname{Arf}\left(g_{k}\right)$ is non-trivial and its restriction to $S^{k} \times\{\bullet\}$ is an orientation preserving homotopy equivalence $S^{k} \times\{\bullet\} \rightarrow S^{k}$;

(iii) If $d=2 \bmod 4$, then there is a map $g_{d}: S^{k} \times S^{d} \rightarrow S^{d}$ such that its Arf invariant $\operatorname{Arf}\left(g_{d}\right)$ is non-trivial and its restriction to $\{\bullet\} \times S^{d}$ is an orientation preserving homotopy equivalence $\{\bullet\} \times S^{d} \rightarrow S^{d}$.

Remark 0.14 (Relation to the Arf-invariant-one-problem). The condition (dii) appearing in Theorem 0.13 (d) implies that the (stable) Arf invariant homomorphism $\operatorname{Arf}_{k}$ of (0.12) is surjective. The problem whether $\operatorname{Arf}_{k}$ is surjective is the famous Arf-invariant-one-problem (see [3]). The map $\Omega_{k}^{\mathrm{fr}} \rightarrow \mathbb{Z} / 2$ is known to be trivial unless $2 k+2$ is of the shape $2^{l}$ for some $l \in \mathbb{Z}$ (see [3]). Hence a necessary condition for $S^{k} \times S^{d}$ to be Borel is that $k$ is odd or that $2 k+2$ is of the shape $2^{l}$ for some $l \in \mathbb{Z}$ and analogously for $d$.

Suppose that the unstable Arf-invariant-map

$$
\operatorname{Arf}_{k, k+d}: \Omega_{k, k+d}^{\mathrm{fr}} \rightarrow \mathbb{Z} / 2 .
$$

is surjective. Then condition (dii) is automatically satisfied by the following argument. Choose a framed manifold $M \subseteq S^{k+d}$ with $\operatorname{Arf}_{k, k+d}([M])=1$. By the Pontrjagin-Thom construction we obtain a map $g_{k}^{\prime}: S^{k+d} \rightarrow S^{k}$ which is transversal to $\{\bullet\} \subseteq S^{k}$ and satisfies $g^{-1}(\{\bullet\})=M$. Now define the desired map $g_{k}$ to be the composition of $g_{k}^{\prime}$ with an appropriate collapse map $S^{k} \times S^{d} \rightarrow S^{k+d}$. So the surjectivity of the unstable Arf invariant map (0.15) implies condition (dii). Of course the surjectivity of (0.15) is in general a stronger condition than the surjectivity of (0.12). The unstable Arf invariant map (0.15) is known to be surjective if $k=2$ and $d \geq 1$. Hence $S^{k} \times S^{d}$ is Borel if $k=2$ and $d \geq 2$ or if $k \geq 2$ and $d=2$.

Now we discuss the following question. How complicated can the homotopy type of Borel manifolds be? In the situation of the Borel and Poincaré Conjectures the homotopy type is determined by the fundamental group and - in the case of homotopy spheres - by the homology groups. Most of our results concerned 
generalized topological space forms (or connected sums of these), spaces which are "close neighbours" of aspherical manifolds resp. homotopy spheres. Besides the products of spheres we have given results concerning other classes of manifolds only in dimension 4. If we concentrate on manifolds with torsionfree fundamental groups (the lens spaces show that even very simple manifolds are in general not Borel for cyclic fundamental groups (see for instance [6, $\S 29$ and $\S 31]$, [26, Section $2.4])$ ), these results in dimension 4 for non-aspherical manifolds concern manifolds with fundamental group $\mathbb{Z}$. Here the fundamental group and the intersection form on $\pi_{2}$, which is a homotopy invariant, determine the homeomorphism type. The following classes of manifolds are natural generalizations of these manifolds.

Problem 0.16 (Classification of certain low-dimensional manifolds). Classify up to orientation preserving homotopy equivalence, homeomorphism (or diffeomorphism in the smooth case) all manifolds in dimension $1 \leq k<n \leq 6$ for which $\pi=\pi_{1}(M)$ is non-trivial and is isomorphic to $\pi_{1}(K)$ for a manifold $K$ of dimension $k \leq 2$ with $\pi_{1}(K) \neq\{1\}$ and whose second homotopy group $\pi_{2}(M)$ vanishes.

Remark 0.17 (Simply-connected case). We have excluded in Problem 0.16 the case $\pi_{1}(K)=\{1\}$ since then a complete answer to this problem is already known. Namely, if $M$ is a 2-connected $n$-dimensional manifold, then $n \geq 3$, it is homotopy equivalent to $S^{3}$ for $n=3$ and it is homeomorphic to $S^{n}$ for $n=4,5$. If $n=6$ and $M$ is a 2-connected smooth manifold, then its oriented homotopy type and its oriented diffeomorphism type are determined by the intersection from on $H_{3}(M)$ (see Wall [37]). This also applies to the topological category by the work of Kirby-Siebenmann [21].

The following results give an almost complete answer to this problem.

Theorem 0.18. (Manifolds appearing in Problem 0.16 of dimension $\leq$ 5). Let $M$ and $K$ be as in Problem 0.16. Let $f: M \rightarrow K=B \pi$ be the classifying map for the $\pi$-covering $\widetilde{M} \rightarrow M$. Suppose $n \leq 5$. Then

(a) Both the oriented homotopy type and the oriented homeomorphism type of $M$ are determined by its second Stiefel-Whitney class. Namely, there is precisely one fiber bundle $S^{3} \rightarrow E \stackrel{p}{\rightarrow}$ K with structure group $S O(4)$ whose second Stiefel-Whitney class agrees with the second Stiefel-Whitney class of $M$ under the isomorphism $H^{2}(f ; \mathbb{Z} / 2): H^{2}(K ; \mathbb{Z} / 2) \stackrel{\cong}{\rightrightarrows} H^{2}(M ; \mathbb{Z} / 2)$. 
There exists an orientation preserving homeomorphism $g: M \rightarrow E$ such that $p \circ g$ and $f$ are homotopic.

In particular the vanishing of the second Stiefel-Whitney class of $M$ implies that there is an orientation preserving homeomorphism $g: M \rightarrow$ $S^{3} \times B \pi$ such that $\mathrm{pr}_{B \pi} \circ g$ and $f$ are homotopic;

(b) The manifold $M$ is never a strong Borel manifold but is always a Borel manifold.

\section{Theorem 0.19. (Manifolds appearing in Problem 0.16 of dimension 6).} Let $M$ and $K$ be as in Problem 0.16. Suppose that $n=6$.

(a) The manifold $M$ is never strongly Borel but always Borel;

(b) Suppose that $w_{2}(M)=0$, or, equivalently, that $M$ admits a Spin-structure. Then both the oriented homotopy type and oriented homeomorphism type of $M$ are determined by the $\mathbb{Z} \pi$-isomorphism class of the intersection form on $H_{3}(\widetilde{M})$.

Given a finitely generated stably free $\mathbb{Z} \pi$-module together with a nondegenerate skew-symmetric $\mathbb{Z} \pi$-form on it, it can be realized as the intersection form of a 6-dimensional manifold having the properties described in Problem 0.16.

One expects that Borel manifolds are an exception. The following results which give necessary conditions for $M$ to be Borel support this intuition.

Theorem 0.20 (A necessary condition for sphere bundles over aspherical manifolds). Let $S^{d} \rightarrow E \stackrel{p}{\rightarrow} K$ be a fiber bundle such that $E$ and $K$ are manifolds and $K$ is aspherical. Assume that there is a map $i: K \rightarrow E$ with $p \circ i \simeq \operatorname{id}_{K}$. Suppose that $d$ is odd and $d \geq 3$ or suppose that $d$ is even and $k \leq d-2$ for $k=\operatorname{dim}(K)$.

Then a necessary condition for $E$ to be a Borel manifold is that $H_{k-4 i}(K ; \mathbb{Q})$ vanishes for all $i \in \mathbb{Z}, i \geq 1$.

Theorem 0.21 (A necessary condition for being a Borel manifold). Let $M$ be a Borel manifold of dimension $n$ with fundamental group $\pi$. Let ho-aut ${ }_{\pi}(M)$ be the set of homotopy classes of orientation preserving self-homotopy equivalences $f: M \rightarrow M$ which induce up to conjugation the identity on the fundamental group. Let $\mathcal{L}(M)_{i} \in H_{4 i}(M ; \mathbb{Q})$ be the $i$-th component of the L-class $\mathcal{L}(M)$ of $M$. 
Then the subset of $\bigoplus_{i \in \mathbb{Z}, i \geq 1} H_{n-4 i}(M ; \mathbb{Q})$

$$
S:=\left\{f_{*}(\mathcal{L}(M) \cap[M])-\mathcal{L}(M) \cap[M] \mid[f] \in \operatorname{ho-aut}_{\pi}(M)\right\}
$$

is an abelian subgroup and the $\mathbb{Q}$-submodule generated by $S$ must contain the kernel of the map induced by the classifying map $c: M \rightarrow B \pi$

$$
c_{*}: \bigoplus_{i \in \mathbb{Z}, i \geq 1} H_{n-4 i}(M ; \mathbb{Q}) \rightarrow \bigoplus_{i \in \mathbb{Z}, i \geq 1} H_{n-4 i}(B \pi ; \mathbb{Q}) .
$$

In particular for every $i \geq 1$ with $\mathcal{L}(M)_{i}=0$ the map $c_{*}: H_{n-4 i}(M ; \mathbb{Q}) \rightarrow$ $H_{n-4 i}(B \pi ; \mathbb{Q})$ must be injective.

We have mentioned that lens spaces are in general not Borel, an indication that torsion in the fundamental group makes Borel less likely. The following result shows that in dimension $4 k+3$ torsion excludes Borel.

Theorem 0.22 (Chang-Weinberger[5]). Let $M^{4 k+3}$ be a closed oriented manifold for $k \geq 1$ whose fundamental group has torsion. Then there are infinitely many pairwise not homeomorphic smooth manifolds which are homotopy equivalent to $M$ (and even simply and tangentially homotopy equivalent to $M$ ) but not homeomorphic to $M$.

Another natural class of manifolds are the homology spheres where surgery gives a necessary and sufficient condition for being strongly Borel if we take the Whitehead torsion into account. Namely, we say that a manifold $M$ is It is called a a strong simple Borel manifold if every orientation preserving simple homotopy equivalence $f: N \rightarrow M$ of manifolds is homotopic to a homeomorphism $h: N \rightarrow M$. If the Whitehead group of $\pi_{1}(M)$ is trivial, the notions of strong Borel manifold and srong simple Borel manifold coincide.

Theorem 0.23 (Homology spheres). Let $M$ be a $n$-dimensional manifold of dimension $n \geq 5$ with fundamental group $\pi=\pi_{1}(M)$.

(a) Let $M$ be an integral homology sphere. Then $M$ is a strong simple Borel manifold if and only if the inclusion $j: \mathbb{Z} \rightarrow \mathbb{Z} \pi$ induces an isomorphism

$$
L_{n+1}^{s}(j): L_{n+1}^{s}(\mathbb{Z}) \stackrel{\cong}{\rightrightarrows} L_{n+1}^{s}(\mathbb{Z} \pi)
$$

on the simple L-groups $L_{n+1}^{s}$; 
(b) Suppose that $M$ is a rational homology sphere and Borel. Suppose that $\pi$ satisfies the Novikov Conjecture in dimension $(n+1)$, i.e., the assembly map $H_{n+1}(B \pi ; \mathbf{L}) \rightarrow L_{n+1}(\mathbb{Z} \pi)$ is rationally injective. Then

$$
H_{n+1-4 i}(B \pi ; \mathbb{Q})=0
$$

for $i \geq 1$ and $n+1-4 i \neq 0$.

The next result and example comes from suggestions of the referee and discussions with Shmuel Weinberger.

Theorem 0.24. $\quad$ (a) Let $f: M \rightarrow N$ be a map of manifolds of dimensions $\geq 5$ which induces an isomorphism on $\pi_{1}$ and on the homology with integral coefficients. Then $M$ is strongly Borel if and only if $N$ is strongly Borel.

(b) Let $M$ be a manifold of dimension $\geq 5$ which is homological aspherical, i.e., its classifying map $M \rightarrow B \pi_{1}(M)$ induces an isomorphism on homology with integral coefficients. Suppose that the Farrell-Jones Conjecture holds for $\pi_{1}(M)$. Then $M$ is strongly Borel.

This leads to the following construction of strongly Borel manifolds. Start with a strongly Borel manifold $M$ of dimension $n \geq 5$. Choose an emdedding $S^{1} \times D^{n-1} \rightarrow M$ which induces an injection on $\pi_{1}$. Choose a high dimensional knot $K \subseteq S^{n}$ with complement $X$ such that the inclusion $\partial X \cong S^{1} \times S^{n-2} \rightarrow X$ is an isomorphism. Put $M^{\prime}=M-\left(S^{1} \times D^{n-1}\right) \cup_{S^{1} \times S^{n-2}} X$. Then $M^{\prime}$ is strongly Borel by Lemma 0.24 (a). If $M$ is aspherical, then $M^{\prime}$ is in general not aspherical.

The paper is organized as follows:

1. On the Structure Set of Certain Topological Manifolds

2. Constructing Self-homotopy Equivalences

3. Sphere Bundles

4. Connected Sums

5. Dimension 3

6. Dimension 4

7. Products of Two Spheres

8. On the Homotopy Type of Certain Low-Dimensional Manifolds

9. On the Classification of Certain Low-Dimensional Manifolds

10. A Necessary Condition for Being a Borel Manifold

11. Integral Homology Spheres

References 
We thank Andrew Ranicki for fruitful discussions about this paper.

\section{On the Structure Set of Certain Topological Manifolds}

We begin with a fundamental criterion for Borel manifolds which follows directly from the definitions.

Theorem 1.1 (Surgery criterion for Borel manifolds). A manifold $M$ is a Borel manifold if and only if the action of the group of homotopy classes of selfhomotopy equivalence $M \rightarrow M$ which induce the identity on the fundamental group up to conjugation on the topological structure set $\mathcal{S}^{\text {top }}(M)$ is transitive, and $M$ is a strong Borel manifold if and only $\mathcal{S}^{\text {top }}(M)$ consists of one element.

Now we determine the topological structure set for certain manifolds. In the sequel we denote by $\mathbf{L}\langle 1\rangle$ the 1-connected cover of the quadratic $L$-theory spectrum $\mathbf{L}$ and by $\mathbf{u}: \mathbf{L}\langle 1\rangle \rightarrow \mathbf{L}$ the canonical map. We get

$$
\pi_{q}(\mathbf{L}\langle 1\rangle)=\left\{\begin{array}{l}
L_{q}(\mathbb{Z}), \text { if } q \geq 1 \\
0, \text { if } q \leq 0
\end{array}\right\}=\left\{\begin{array}{l}
\mathbb{Z}, \text { if } q \geq 4, q=0 \bmod 4 \\
\mathbb{Z} / 2, \text { if } q \geq 2, q=2 \bmod 4 \\
0 \quad \text { otherwise }
\end{array}\right.
$$

Theorem 1.2. Let $M$ be an $n$-dimensional manifold for $n \geq 5$. Let $K$ be an aspherical k-dimensional manifold with fundamental group $\pi$. Suppose that the Farrell-Jones Conjecture for algebraic $K$ - and L-theory holds for $\pi$. Let $f: M \rightarrow$ $K$ be a 2-connected map. Suppose that we can choose a map $i: K \rightarrow M$ such that $f \circ i$ is homotopic to the identity.

(a) The homomorphism

$$
H_{m}\left(\operatorname{id}_{K} ; \mathbf{u}\right): H_{m}(K ; \mathbf{L}\langle 1\rangle) \rightarrow H_{m}(K ; \mathbf{L})
$$

is bijective for $m \geq k+1$ and injective for $k=m$;

(b) The exact topological surgery sequence for $M$ yields the short split-exact sequence

$$
0 \rightarrow \mathcal{S}^{\text {top }}(M) \stackrel{\sigma_{n}}{\longrightarrow} H_{n}(M ; \mathbf{L}\langle 1\rangle) \stackrel{H_{n}(f ; \mathbf{L}\langle 1\rangle)}{\longrightarrow} H_{n}(K ; \mathbf{L}\langle 1\rangle) \rightarrow 0 .
$$

In particular we get an isomorphism

$$
\bar{\sigma}_{n}: \mathcal{S}^{\text {top }}(M) \stackrel{\cong}{\rightrightarrows} H_{n}(i: K \rightarrow M ; \mathbf{L}\langle 1\rangle) .
$$


Proof. (a) Let $\mathbf{E}$ be the homotopy fiber of $\mathbf{u}$. Hence we have a fibration of spectra

$$
\mathbf{E} \rightarrow \mathbf{L}\langle 1\rangle \stackrel{\mathbf{u}}{\rightarrow} \mathbf{L}
$$

which induces a long exact sequence

$$
\begin{aligned}
\ldots \rightarrow H_{m+1}(K ; \mathbf{L}\langle 1\rangle) \rightarrow H_{m+1}(K ; \mathbf{L}) & \rightarrow H_{m}(K ; \mathbf{E}) \\
& \rightarrow H_{m}(K ; \mathbf{L}\langle 1\rangle) \rightarrow H_{m}(K ; \mathbf{L}) \rightarrow \ldots
\end{aligned}
$$

Since $\pi_{q}(\mathbf{E})=0$ for $q \geq 0$, an easy spectral sequence argument shows that $H_{m}(K ; \mathbf{E})=0$ for $m \geq k$. Hence the map

$$
H_{m}\left(\operatorname{id}_{K} ; \mathbf{u}\right): H_{m}(K ; \mathbf{L}\langle 1\rangle) \rightarrow H_{m}(K ; \mathbf{L})
$$

is bijective for $m \geq k+1$ and injective for $k=m$.

(b) There is an exact sequence of abelian groups called algebraic surgery exact sequence [33, Definition 15.19 on page 169].

$$
\begin{aligned}
\ldots \stackrel{\sigma_{n+1}}{\longrightarrow} H_{n+1}(X ; \mathbf{L}\langle 1\rangle) \stackrel{A_{n+1}}{\longrightarrow} L_{n+1}\left(\mathbb{Z} \pi_{1}(X)\right) \stackrel{\partial_{n+1}}{\longrightarrow} \\
\quad \mathcal{S}^{\text {top }}(X) \stackrel{\sigma_{n}}{\longrightarrow} H_{n}(X ; \mathbf{L}\langle 1\rangle) \stackrel{A_{n}}{\longrightarrow} L_{n}\left(\mathbb{Z} \pi_{1}(X)\right) \stackrel{\partial_{n}}{\longrightarrow} \ldots
\end{aligned}
$$

which is defined for every simplicial connected complex $X$ and natural in $X$. It agrees for $X$ a $n$-dimensional manifold for $n \geq 5$ with the Sullivan-Wall geometric exact surgery sequence [33, Theorem 18.5 on page 198]. Notice that by assumption $\mathrm{Wh}_{i}\left(\pi_{1}(K)\right)$ vanishes for $i \leq 1$ so that we can ignore any decorations in the sequel. The following diagram commutes for all $m$

$$
\begin{array}{cc}
H_{m}(M ; \mathbf{L}\langle 1\rangle) & \stackrel{A_{m}}{\longrightarrow} L_{m}(\mathbb{Z} \pi) \\
H_{m}(f ; \mathbf{L}\langle 1\rangle) \downarrow & \text { id } \mid \cong \\
H_{m}(K ; \mathbf{L}\langle 1\rangle) & \stackrel{A_{m}}{\longrightarrow} L_{m}(\mathbb{Z} \pi) \\
H_{m}\left(\operatorname{id}_{K} ; \mathbf{u}\right) \downarrow & \text { id } \mid \cong \\
H_{m}(K ; \mathbf{L}) & \stackrel{A_{m}}{\cong} L_{m}(\mathbb{Z} \pi)
\end{array}
$$

The existence of the map $i$ with $f \circ i \simeq \operatorname{id}_{K}$ ensures that $H_{m}(f ; \mathbf{L}\langle 1\rangle)$ is surjective. Now the claim follows from assertion (a) and the exact sequence (1.3) for $X=$ $M$.

Theorem 0.24 follows now from Theorem 1.1, Theorem 1.2 (b), the exact sequence (1.3) and the Atiyah-Hirzebruch spectral sequence. 
Theorem 1.4. Let $K$ be an aspherical $k$-dimensional manifold with fundamental group $\pi$. Suppose that the Farrell-Jones Conjecture for algebraic $K$ - and $L$-theory holds for $\pi$. Let $d$ be an integer with $d \geq 2$ and $d+k \geq 5$. Consider a fiber bundle $S^{d} \rightarrow E \stackrel{p}{\rightarrow} K$ such that $E$ is oriented. Suppose that there exists a map $i: K \rightarrow E$ with $p \circ i=\mathrm{id}$.

Then $i: K \rightarrow E$ is an embedding of topological manifolds and we obtain an isomorphism of abelian groups

$$
\mathcal{S}^{\text {top }}(E) \stackrel{\cong}{\longrightarrow} H_{k}(K ; \mathbf{L}\langle 1\rangle) .
$$

It sends under the identification of $H_{k}(K ; \mathbf{L}\langle 1\rangle)$ with the set $\mathcal{N}(K) \cong[K, G / T O P]$ of normal surgery problems with $K$ as reference space an element $f: M \rightarrow E$ to the following surgery problem: By changing $f$ up to homotopy we can arrange that $f$ is transverse to $i: K \rightarrow E$. Let $g: N=f^{-1}(i(K)) \rightarrow K$ be the map of manifolds of degree 1 induced by $f$ and $i^{-1}: i(K) \rightarrow K$. By transversality we obtain a bundle map $\bar{g}: \nu(N, M) \rightarrow \nu(i)$ covering $g$. Choose a vector bundle $\xi \rightarrow M$ and a bundle map $\bar{f}: \nu(M) \rightarrow \xi$ covering $f: M \rightarrow E$. Then $g$ is covered by the bundle map

$$
\left.\bar{g} \oplus \bar{f}\right|_{N}: \nu(N)=\left.\nu(N, M) \oplus \nu(M)\right|_{N} \rightarrow \nu(i) \oplus i^{*} \xi,
$$

and these data give the desired surgery problem with target $K$.

Proof. See [38, Chapter 11], [33, pages 257-260].

Theorem 1.5. Let $K$ be an aspherical $k$-dimensional manifold with fundamental group $\pi$. Consider a fiber bundle $S^{d} \rightarrow E \stackrel{p}{\rightarrow} K$ with $d \geq 1$. Suppose that the Farrell-Jones Conjecture for algebraic $K$ - and L-theory holds for $\pi$. Then:

(a) If $k=2$ and $d \geq 3$, then

$$
\mathcal{S}^{\text {top }}(E) \cong L_{2}(\mathbb{Z}) \cong \mathbb{Z} / 2
$$

(b) If $k=3$ and $d \geq 4$ or if $k=3, d=2,3$ and there is a map $i: K \rightarrow E$ with $p \circ i=\operatorname{id}_{K}$, then

$$
\mathcal{S}^{\text {top }}(E) \cong H_{1}\left(K ; L_{2}(\mathbb{Z})\right) \cong H_{1}(K ; \mathbb{Z} / 2) ;
$$

(c) If $k=1$ and $d \geq 3$, then

$$
\mathcal{S}^{\text {top }}(E)=0
$$


Proof. We first prove the claim in the case $k+d \geq 5$. Since $K$ is $k$-dimensional and $S^{d}$ is $(d-1)$-connected, we can find a map $i: K \rightarrow E$ such that $p \circ i$ is homotopic to $\mathrm{id}_{K}$ provided $d>k$. By the homotopy lifting property we can arrange that $p \circ i$ is $\operatorname{id}_{K}$. By assumption such a map $i$ exists also in the remaining case $k=3$ and $d=2,3$. Now the claim follows from Theorem 1.4 and an easy computation with the Atiyah-Hirzebruch spectral sequence. Thus we have proven assertions (a) and (b) and for assertion (c) only the case $k=1$ and $d=3$ remains open. Then $\pi_{1}(E)$ is $\mathbb{Z}$ which is a good fundamental group in the sense of Freedman [17]. Hence topological surgery works also in this dimension 4 and the same argument which gives the claim for $k=1$ and $k+d \geq 5$ works also for $k=1$ and $k+d=4$.

\section{Constructing Self-homotopy Equivalences}

In this section we describe a certain construction of self-homotopy equivalences. It will be used to show that the action of the group of orientation preserving homotopy equivalences $E \rightarrow E$ which induce the identity on the fundamental group up to conjugation on $\mathcal{S}^{\text {top }}(E)$ is transitive for certain manifolds $E$.

Suppose we are given the following data:

- Let $K$ and $E$ be manifolds with $\operatorname{dim}(K)=k$ and $\operatorname{dim}(E)=k+d$ for $k, d \geq 2$;

- An embedding $i_{K}: K \rightarrow E$;

- A map $\phi: S^{d} \rightarrow E$ which is transversal to $i_{K}: K \rightarrow E$ and the intersection of the images $\operatorname{im}(\phi)$ and $\operatorname{im}\left(i_{K}\right)$ consists of precisely one point $e_{0} \in E$;

- Let $M$ be a manifold of dimension $k$ together with an embedding $i_{M}: M \rightarrow$ $S^{k+d}$ and a framing of the normal bundle $\mu\left(i_{M}\right)$;

- $k+d \geq 5$ or $E$ is simply connected.

Fix an embedding $j_{K}: D^{k} \rightarrow K$ with $i_{K} \circ j_{K}(0)=e_{0}$. Since $D^{k}$ is contractible, we can choose a disk bundle map

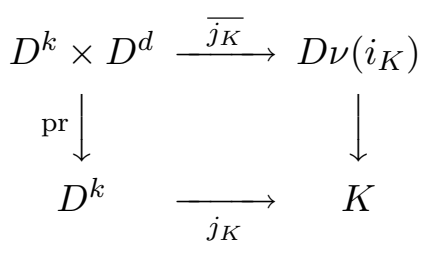


which is fiberwise a homeomorphism. Choose an embedding $j_{M}: D^{k} \rightarrow M$. Since $D^{k}$ is contractible, we can choose a disk bundle map

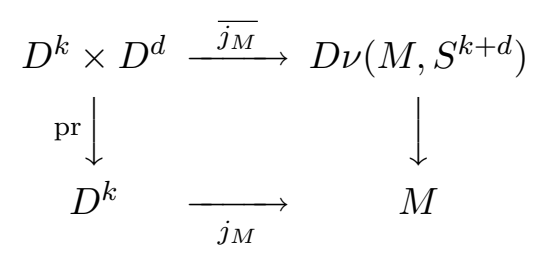

which is fiberwise a homeomorphism. Using a tubular neighborhood, we will also regard $\overline{j_{K}}$ as an embedding $D^{k} \times D^{d} \rightarrow E$ extending $j_{K}: D^{k} \rightarrow E$ and $\overline{j_{M}}$ as an embedding $D^{k} \times D^{d} \rightarrow S^{k+d}$ extending $j_{M}: D^{k} \rightarrow S^{k+d}$.

In the sequel let $j_{S^{d}}: D^{d} \rightarrow S^{d}$ be the obvious embedding given by the lower hemisphere. Recall that $\phi$ and $i_{K}$ are transversal to one another and the intersection of their images consists of the point $e_{0}$. We can assume without loss of generality that $\phi \circ j_{S^{d}}(0)=e_{0}=j_{K}(0)$ holds. Now we can thicken $\phi$ to a map $\bar{\phi}: D^{k} \times S^{d} \rightarrow E$ such that the composite

$$
D^{k} \times D^{d} \stackrel{\text { id } \times j_{S^{d}}}{\longrightarrow} D^{k} \times S^{d} \stackrel{\bar{\phi}}{\rightarrow} E
$$

agrees with the embedding $\overline{j_{K}}: D^{k} \times D^{d} \rightarrow E$ and the intersection of the image of $\bar{\phi}$ and of $D \nu\left(i_{K}\right)$ considered as subset of $E$ is the image of $\overline{j_{K}}: D^{k} \times D^{d} \rightarrow E$.

The Pontrjagin-Thom construction applied to $i_{M}: M \rightarrow S^{d-k}$ together with the given framing on $M$ yields a map PT: $S^{k+d} \rightarrow S^{d}$ such that PT is transversal to $i_{S^{d}}(0) \in S^{d}$, the preimage of $i_{S^{d}}(0) \in S^{d}$ is just $M$ and the bundle map given by transversality $\nu\left(i_{M}\right) \rightarrow \nu\left(\left\{i_{S^{d}}(0)\right\} \subseteq S^{d}\right)$ is just the given framing. We can arrange by shrinking $D^{d}$ that the composition of PT with $\overline{j_{M}}: D^{k} \times D^{d} \rightarrow S^{k+d}$ agrees with the composite

$$
D^{k} \times D^{d} \stackrel{\mathrm{pr}}{\longrightarrow} D^{d} \stackrel{j_{S^{d}}}{\longrightarrow} S^{d} .
$$

Choose a map $c: S^{k+d} \rightarrow D^{k}$ such that its composite with the embedding $\overline{j_{M}}: D^{k} \times D^{d} \rightarrow S^{k+d}$ is the projection pr: $D^{k} \times D^{d} \rightarrow D^{k}$, it is transversal to $0 \in D^{k}$ and the preimage of 0 is an embedded $S^{d}$. Such a map can be constructed from the map $S^{k+d}=S^{d} * S^{k-1} \rightarrow\{\bullet\} * S^{k-1}=D^{k}$.

Now consider the composite

$$
\alpha: S^{k+d} \stackrel{c \times \mathrm{PT}}{\longrightarrow} D^{k} \times S^{d} \stackrel{\bar{\phi}}{\longrightarrow} E .
$$


Its composition with the embedding $\overline{j_{M}}: D^{k} \times D^{d} \rightarrow S^{k+d}$ agrees with the embed$\operatorname{ding} \overline{j_{K}}: D^{k} \times D^{d} \rightarrow E$. It is transverse to $i_{K}: K \rightarrow E$ such that the preimage of both $i_{K}(K)$ and of $i_{K} \circ j_{K}\left(D^{k}\right)$ is $M$ and the bundle map given by transversality from $\nu\left(M, S^{k+d}\right) \rightarrow i_{K}^{*} \nu(i)$ is compatible up to isotopy with the given framing of $\nu\left(M ; S^{k+d}\right)$ and some framing of the bundle $i_{K}^{*} \nu(i)$ over $D^{k}$.

In the sequel we consider the connected sum $E \# S^{d+k}$ with respect to the two embeddings $\overline{j_{M}}: D^{k} \times D^{d} \rightarrow S^{k+d}$ and $\overline{j_{K}}: D^{k} \times D^{d} \rightarrow E$. By construction the identity id: $E \rightarrow E$ and the map $\alpha: S^{k+d} \rightarrow E$ fit together and yield a map id $\# \alpha: E \# S^{k+d} \rightarrow E$.

We claim that this map is a homotopy equivalence. Choose a point $x \in E$ which not contained in the images of $\overline{j_{K}}: D^{k} \times D^{d} \rightarrow E$ and of $\bar{\phi}: D^{k} \times S^{d} \rightarrow E$. Then the preimage of $x$ under id \# $\alpha$ is $x$ and the map id \# $\alpha$ induces the identity on a neighborhood of $x$. This implies that id $\# \alpha$ has degree one. The inclusions of $E-\operatorname{im}\left(\overline{j_{K}}\right)$ into both $E$ and $E \# S^{k+d}$ are $(k+d-1)$-connected. Since id \# $\alpha$ induces the identity on $E-\operatorname{im}\left(\overline{j_{K}}\right)$, the homomorphisms $\pi_{j}$ (id $\left.\# \alpha\right)$ are bijective for $j \leq k+d-2$. By assumption we have $k+d \geq 5$ or we have $k+d=4$ and $\pi_{1}(E)=\{1\}$. Now we conclude from Poincaré duality that id $\# \alpha: E \# S^{k+d} \rightarrow E$ is a homotopy equivalence.

Obviously we can find a homeomorphism $\beta: E \rightarrow E \# S^{k+d}$ such that the composite $\alpha \circ \beta$ is the identity outside the image of $\overline{j_{K}}: D^{k} \times D^{d} \rightarrow E$.

The map id \# $\alpha: E \# S^{k+d} \rightarrow E$ is transversal to $i_{K}: K \rightarrow E$. The preimage of $i_{K}$ is the connected sum $K \# M$, which is taken with respect to the embeddings $j_{M}: D^{k} \rightarrow M$ and $j_{K}: D^{k} \rightarrow K$. This map has degree one and is covered by bundle data due to transversality. The resulting normal map with target $K$ agrees with the connected sum of the normal map id: $K \rightarrow K$ and the normal map $M \rightarrow S^{k}$ given by the collapse map of degree one and the bundle data coming from the given framing on $M$.

Now additionally suppose that the given map $\phi: S^{d} \rightarrow E$ is an embedding. (It is automatically a local embedding near the intersection point with $K$ by transversality but a priori not a global embedding). Then also the map $\bar{\phi}: D^{k} \times$ $S^{d} \rightarrow E$ can be chosen to be an embedding. It is not hard to check that the map id $\# \alpha: E \# S^{k+d} \rightarrow E$ is transversal to $\phi$ and the corresponding surgery problem is given by a homeomorphism $(\mathrm{id} \# \alpha)^{-1}\left(\alpha\left(S^{d}\right)\right) \stackrel{\cong}{\longrightarrow} S^{d}$ covered by an 
isomorphism of the normal bundles. In particular this surgery problem represents the trivial element in $\mathcal{N}\left(S^{d}\right)$.

Next we explain the maps in the following diagram

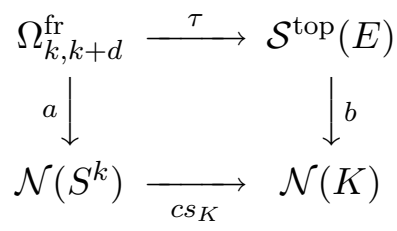

Recall that we denote by $\Omega_{k, k+d}^{\mathrm{fr}}$ the set of bordism classes of $k$-dimensional manifolds $M$ together with an embedding $M \subseteq \mathbb{R}^{k+d}$ and an (unstable) framing of its normal bundle $\nu\left(M \subseteq \mathbb{R}^{k+d}\right)$. The map $\tau$ is given by the construction above which assigns to $[M] \in \Omega_{k, k+d}^{\mathrm{fr}}$ the element in the structure set given by $\alpha: E \# S^{k+d} \rightarrow E$. The map $a$ sends a framed $k$-dimensional manifold $M$ to the normal map given by the collapse map $c: M \rightarrow S^{k}$ covered by stable bundle map from $\nu(M)$ to the trivial bundle over $S^{k}$ given by the framing. The map $c s_{K}$ is given by taking the connected sum of a surgery problem with target $S^{k}$ with the one given by the identity $\operatorname{id}_{K}: K \rightarrow K$. The map $b$ sends the class of a homotopy equivalence $f: N \rightarrow E$ to the surgery problem with underlying map $f^{-1}\left(i_{K}(K)\right) \rightarrow K$ after making $f$ transversal to $i_{K}: K \rightarrow E$.

We have shown

Theorem 2.2. $\quad$ (a) The diagram 2.1 commutes;

(b) Each element in the image of $\tau$ is represented by a self-homotopy equivalence $E \rightarrow E$ which induces for some embedded disk $D^{n+k} \subseteq E$ the identity id: $E-D^{n+k} \rightarrow E-D^{n+k}$ and in particular induces up to conjugation the identity on the fundamental groups;

(c) Suppose additionally that the given map $\phi: S^{d} \rightarrow E$ is an embedding. Let $b^{\prime}: \mathcal{S}^{\text {top }}(E) \rightarrow \mathcal{N}\left(S^{d}\right)$ be the map given by making a homotopy equivalence $f: N \rightarrow E$ transversal to $\phi$. Then the composite

$$
\Omega_{k, k+d}^{\mathrm{fr}} \stackrel{\tau}{\rightarrow} \mathcal{S}^{\mathrm{top}}(E) \stackrel{b^{\prime}}{\rightarrow} \mathcal{N}\left(S^{d}\right)
$$

is trivial;

\section{SPHERE Bundles}

In this section we prove Theorem 0.6 and Theorem 0.8 . 
Proof. We begin with Theorem 0.6. It follows from Theorem 1.5, Theorem 2.2 (a) and Theorem 1.1 since the 2-dimensional torus with an appropriate framing yields an element $\Omega_{2,2+d}^{\mathrm{fr}}$ whose image under the Arf invariant map $\Omega_{2,2+d}^{\mathrm{fr}} \rightarrow \mathbb{Z} / 2$ is non-trivial.

Proof. Next we prove Theorem 0.8. Assertion (a) follows from Theorem 1.5 (b) and Theorem 1.1.

To prove Assertion (b) and Assertion (c) we use the modified surgery theory from [22], to which we refer for notation. Let $f: M \rightarrow K \times S^{d}$ be a homotopy equivalence. The normal ( $d-2)$-type of $M$ is $K \times B T O P\langle d-1\rangle$, where $B T O P\langle d-$ $1\rangle$ is the $(d-1)$-connected cover of the classifying space of topological vector bundles BTOP. The reason is that the restriction of the normal bundle to the $(d-1)$-skeleton, which is homotopy equivalent to $K$ is determined by $w_{2}(\nu(M))$. But the Stiefel-Whitney classes are homotopy invariants, and so $w_{2}(\nu(M))=0$. If the restriction of the normal bundle to the $(d-1)$-skeleton is trivial, we obtain a normal $(d-1)$-smoothing of $M$ in $K \times B T O P\langle d-1\rangle$ by choosing a map from $M$ to $K$ inducing $f_{*}$ on $\pi_{1}$ up to conjugation and by choosing a framing on the $(d-1)$-skeleton. Again we use that the $(d-1)$-skeleton is homotopy equivalent to $K$. Thus a framing on the restriction of the normal bundle to $K$, considered as the $(d-1)$-skeleton of $M$, together with $f_{*}: \pi_{1}(M) \rightarrow \pi_{1}(K)$ determine a normal $(d-1)$-smoothing of $M$ in $K \times B T O P\langle d-1\rangle$. Since $d \geq 7$, we conclude that $(d-1)$ is larger than half the dimension of $K \times S^{d}$. Thus by [22, Theorem 3] and the remark before it and by [22, Theorem 4$]$ and the remark before it the obstruction for replacing a normal bordism between $K \times S^{d}$ and $M$ considered as elements of $\Omega_{d+3}^{T O P\langle d-1\rangle}(K)$ by a $s$-cobordism takes values in the Wall group $L_{d+4}\left(\pi_{1}(K)\right)$. Here we recall that since we assume the Farrell-Jones Conjecture for $K$-theory we don't need to take the Whitehead torsion into account. From Theorem 1.2 we know that the $L$-group acts trivially on the structure set which implies that the obstruction in our situation vanishes since the action in our situation factors through the structure set.

Summarizing these considerations we see that $M$ is homeomorphic to $K \times S^{d}$ inducing $f_{*}$ on $\pi_{1}$ up to conjugation if and only if after choosing the framing on $K$, considered as the $(d-1)$-skeleton of $M$, appropriately the two manifolds are bordant in $\Omega_{d+3}^{T O P\langle d-1\rangle}(K)$. The different choices of a lift of the normal Gauss map of $K \times S^{d}$ to $B T O P\langle d-1\rangle$ correspond to the choice of framings on $K$ and so 
$K \times S^{d}$ is null-bordant for all choices of lifts. This implies the following criterion which we will use below: $M$ is homeomorphic to $K \times S^{d}$ inducing $f_{*}$ on $\pi_{1}$ up to conjugation if and only if $M$ for one (and then for all) topological framings on $K$, considered as the $(d-1)$ - skeleton of $M$, together with the map to $K$ given by $f_{*}$ on $\pi_{1}$ represents the zero class in $\Omega_{d+3}^{T O P\langle d-1\rangle}(K)$.

Next we determine the bordism groups $\Omega_{k}^{T O P\langle d-1\rangle}$ for $d \leq k \leq d+3$. If $N$ together with a lift of the normal Gauss map represents an element in this group we can make it highly connected by surgery. If $k$ is odd we can even pass to a homotopy sphere which by the topological Poincaré Conjecture is null-bordant. Thus $\Omega_{k}^{T O P\langle d-1\rangle}=0$ for $k$ odd (in our range). If $k$ is even, the obstruction for passing to a homotopy sphere is the signature, if $k=0 \bmod 4$ and the Arf invariant, if $k=2 \bmod 4$. Since there exist almost parallelizable manifolds with signature resp. Arf invariant non-trivial, the bordism groups $\Omega_{k}^{T O P\langle d-1\rangle}$ are $\mathbb{Z}$, classified by the signature, if $k=0 \bmod 4$ and $\mathbb{Z} / 2$ detected by the Arf invariant, if $k=2 \bmod 4$.

Now we are ready to prove assertion (b). The Atiyah-Hirzebruch spectral sequence implies for $d=3 \bmod 4$

$$
\Omega_{d+3}^{T O P\langle d-1\rangle}(K) \cong \Omega_{d+3}^{T O P\langle d-1\rangle} \oplus H_{2}(K ; \mathbb{Z}) \cong \mathbb{Z} / 2 \oplus H_{2}(K ; \mathbb{Z}) .
$$

Here the first component is determined by the Arf invariant. For the detection of second component we note that $H_{2}(K ; \mathbb{Z})$ is isomorphic to $\mathbb{Z}$, and so we can pass to $\mathbb{Q}$-coefficients. But then the second component is determined by the higher signatures. Since the Farrell-Jones Conjecture implies the Novikov Conjecture, the higher signatures of $M \rightarrow K$ agree with the ones of $K \times S^{d} \rightarrow K$ and hence vanish. For the Arf invariant we note that we one can interpret it as an Arf invariant of a quadratic from given by a Wu-orientation [3, Theorem 3.2] and so it is also a homotopy invariant. It vanishes for $K \times S^{d} \rightarrow K$ and hence also for $M \rightarrow K$. Thus $M \rightarrow K$ is null-bordant in $\Omega_{d+3}^{T O P\langle d-1\rangle}(K)$. Hence an application of the criterion above finishes the proof of assertion (b).

To prove assertion (c) we again use the Atiyah-Hirzebruch spectral sequence to show

$$
\Omega_{d+3}^{T O P\langle d-1\rangle}(K) \cong H_{1}(K ; \mathbb{Z} / 2) \oplus H_{3}(K ; \mathbb{Z}) .
$$

We suppose that for $d=0 \bmod 4$ and $d \geq 8$ we have $H_{1}(K ; \mathbb{Z} / 2) \neq 0$. Let $g: S^{1} \rightarrow K$ be a map representing a non-trivial element in $H_{1}(K ; \mathbb{Z} / 2)$. We 
consider the connected sum of $K \times S^{d}$ and $S^{1} \times A$, where $A$ is the framed highly connected topological manifolds with Arf invariant 1 (obtained from plumbing two disk bundles of the tangent bundle of the sphere). So we get normal degree one map

$$
\operatorname{id}_{K \times S^{d}} \#\left(g \circ p_{1}\right):\left(K \times S^{d}\right) \#\left(S^{1} \times A\right) \rightarrow K \times S^{d} .
$$

After composition with the projection $K \times S^{d} \rightarrow K$ we obtain an element in $\Omega_{d+3}^{T O P\langle d-1\rangle}(K)=H_{1}(K ; \mathbb{Z} / 2) \oplus H_{3}(K ; \mathbb{Z})$. The element is non-trivial since its component $H_{1}(K ; \mathbb{Z} / 2)$ is the element represented $g$. This follows from the product structure of the Atiyah-Hirzebruch spectra sequence.

By the following sequence of surgeries we replace this map by a homotopy equivalence $f: M \rightarrow K \times S^{d}$. Since $\pi_{1}\left(\left(K \times S^{d}\right) \#\left(S^{1} \times A\right)\right) \cong \pi_{1}\left(K \times S^{d}\right) * \mathbb{Z}$, we can do one 1-dimensional surgery to make the map an isomorphism with out changing the homology groups of the universal coverings up to the middle dimension. Since $A$ is $(d / 2+1)$-connected and $H_{d / 2+1}(A)$ is $\mathbb{Z} \oplus \mathbb{Z}$, the map is already highly connected and the kernel in the dimension $(d / 2+1)$ on the level of the universal coverings is a free $\mathbb{Z}\left[\pi_{1}\left(K \times S^{d}\right)\right]$-module of rank two. Thus by two further surgeries we can obtain the desired homotopy equivalence $f: M \rightarrow K \times S^{d}$. It represents after composition with the projection $K \times S^{d} \rightarrow K$ a non-trivial class in $\Omega_{d+3}^{T O P\langle d-1\rangle}(K)$. By the criterion mentioned above there is no homeomorphism from $M$ to $K \times S^{d}$ inducing the same map on $\pi_{1}$ up to conjugation. Thus in this situation $K \times S^{d}$ is not Borel.

\section{Connected Sums}

In this section we prove Theorem 0.9.

Proof. The main ingredient is the result of Cappell [4, Theorem 0.3] together with the recent improvement of Davis and Connolly [7] that under our assumptions for every homotopy equivalence $f: N \rightarrow M_{1} \# M_{2}$ there are $n$-dimensional manifolds $N_{0}$ and $N_{1}$ together with orientation preserving homotopy equivalences $f_{0}: N_{0} \rightarrow M_{0}$ and $f_{1}: N_{1} \rightarrow M_{1}$ and an orientation preserving homeomorphism $h: N_{0} \# N_{1} \rightarrow N$ such that $f \circ h$ is homotopic to $f_{0} \# f_{1}$. Now the claim follows from Theorem 1.1. 


\section{Dimension 3}

Next we prove Theorem 0.7.

Proof. If $M$ and $N$ are prime Haken 3-manifolds, then every homotopy equivalence $\pi_{1}(M) \rightarrow \pi_{1}(N)$ is homotopic to a homeomorphism. This is a result of Waldhausen (see for instance [20, Lemma 10.1 and Corollary 13.7]). Turaev [35] has extended this result to showing that a simple homotopy equivalence between 3-manifolds with torsionfree fundamental group is homotopic to a homeomorphism provided that Thurston's Geometrization Conjecture for irreducible 3-manifolds with infinite fundamental group and the 3-dimensional Poincaré Conjecture are true. This statement remains true if one replaces simple homotopy equivalence by homotopy equivalence. This follows from the fact explained below that the Whitehead group of the fundamental group of a 3-manifold vanishes provided that Thurston's Geometrization Conjecture for irreducible 3-manifolds with infinite fundamental group is true.

The vanishing of the Whitehead group is proved for Haken manifolds in Waldhausen [36, Section 19]. In order to prove it for prime 3-manifolds it remains to treat closed hyperbolic manifolds and closed Seifert manifolds. These cases are consequences of [13, Theorem 2.1 on page 263 and Proposition 2.3]. Now apply the fact that the Whitehead group of a free amalgamated product is the direct sum of the Whitehead groups.

Every 3-manifold is a generalized topological space form by the following argument. Suppose that $\pi_{1}(M)$ is finite. Then the universal covering is a closed simply connected 3-manifold and hence homotopy equivalent to $S^{3}$. Suppose that $\pi_{1}(M)$ is infinite. Then the universal covering is a non-compact 3-manifold and hence homotopy equivalent to a 2-dimensional $C W$-complex. This implies that the second homology group of $\widetilde{M}$ is a subgroup of a free abelian group, namely the second chain module of the cellular chain complex of $\widetilde{M}$, and hence free as abelian group and that all other homology groups of $\widetilde{M}$ are trivial.

\section{Dimension 4}

Here we prove Theorem 0.11 . 
Proof. (a) Hambleton-Kreck [19, Theorem C] show that the homeomorphism type (including a reference map $M \rightarrow B \pi_{1}(M)$ ) is determined for a 4-manifold with Spin structure and finite cyclic fundamental group by the intersection form on $M$. Hence such a manifold is Borel.

Here we use the result taken from $[18,10.2 \mathrm{~B}]$ that for a 4 -manifold $M$ with Spin structure its signature is divisible by 16 and its Kirby Siebenmann invariant can be read off from the signature by $\operatorname{ks}(M)=\operatorname{sign}(M) / 8 \bmod 2$ and hence is an invariant of its oriented homotopy type.

Now suppose that $M$ is simply connected and admits no Spin structure. Then there exists another simply connected 4-manifold $* M$ with the same intersection form but different Kirby Siebenmann invariant (see [18, 10.1]. In particular $M$ and $* M$ are not homeomorphic but they are oriented homotopy equivalent by [28].

(b) The Borel Conjecture is true for a flat smooth Riemannian 4-manifold (see [10, page 263]. Hence such a manifold is strongly Borel. The manifold $S^{1} \times S^{3}$ is strongly Borel by Theorem 0.13 (a).

The claim about the connected sum $M \# N$ follows from the version of Theorem 0.9 for dimension 4 whose proof goes through in dimension 4 since the fundamental group of $M \# N$ is good in the sense of Freedman [17].

\section{Products of Two Spheres}

In this section we give the proof of Theorem 0.13

Proof. (a) If $k+d=2$, then the property strongly Borel follows from classical results.

If $k=1$ and $d \geq 3$ or $k \geq 3$ and $d=1$, the claim follows from Theorem 1.5 and Theorem 1.1 since the Farrell-Jones Conjecture is known to be true for $\pi=\mathbb{Z}$.

It remains to treat the case $k, d \geq 2$ and $k+d \geq 4$. Then $S^{k} \times S^{d}$ is simplyconnected. The structure set can be computed by

$$
a_{1} \times a_{2}: \mathcal{S}^{\text {top }}\left(S^{k} \times S^{d}\right) \stackrel{\cong}{\rightarrow} L_{k}(\mathbb{Z}) \oplus L_{d}(\mathbb{Z}),
$$


where $a_{1}$ and $a_{2}$ respectively send the class of an orientation preserving homotopy equivalence $f: M \rightarrow S^{k} \times S^{d}$ to the surgery obstruction of the surgery problem with target $S^{k}$ and $S^{d}$ respectively which is obtained from $f$ by making it transversal to $S^{k} \times\{\bullet\}$ and $\{\bullet\} \times S^{d}$ respectively. The proof of this claim is analogous to the one of Theorem 1.4 (see also [33, Example 20.4 on page 211]). Hence the structure set is trivial if and only if both $k$ and $d$ are odd. Now the claim follows from Theorem 1.1. Notice that we can apply surgery theory also to the 4-dimensional manifold $S^{2} \times S^{2}$ since its fundamental group is good in the sense of Freedman [17].

(b) This follows from Theorem 1.5 (c) and Theorem 1.1 in the case $k \geq 3$. It remains to treat the case $d=2$.

Suppose that $S^{1} \times S^{2}$ is Borel. Let $N$ be a homotopy 3 -sphere. There exists an orientation preserving homotopy equivalence $f: S^{1} \times S^{2} \# N \rightarrow S^{1} \times S^{2}$. Since $S^{1} \times S^{2}$ is Borel by assumption, we can choose $f$ to be a homeomorphism. By the uniqueness of the prime decomposition $N$ must be homeomorphic to $S^{3}$. Hence the 3-dimensional Poincaré Conjecture is true.

Now suppose that the 3-dimensional Poincaré Conjecture is true. By the Sphere Theorem [20, Theorem 4.3], an irreducible (closed) 3-manifold is aspherical if and only if it has infinite fundamental group. A prime 3 -manifold is either irreducible or is homeomorphic to $S^{1} \times S^{2}$ [20, Lemma 3.13]. Hence for a prime 3-manifold $M$ with infinite fundamental group the following statements are equivalent: i.) $M$ is irreducible, ii.) $M$ is aspherical, iii.) $\pi_{1}(M)$ is not isomorphic to $\mathbb{Z}$ and iv.) $M$ is not homeomorphic to $S^{1} \times S^{2}$. Now the prime decomposition of 3-manifolds implies that any 3-manifold with fundamental group $\mathbb{Z}$ is homeomorphic to $S^{1} \times S^{2}$. Hence it suffices to show that any orientation preserving homotopy equivalence $f: S^{1} \times S^{2} \rightarrow S^{1} \times S^{2}$ is homotopic to a homeomorphism.

Since there exists orientation reversing homeomorphisms $S^{1} \rightarrow S^{1}$ and $S^{2} \rightarrow$ $S^{2}$, it suffices to treat the case, where $f$ induces the identity on $\pi_{1}\left(S^{1} \times S^{2}\right)$. Then one can change $f$ up to homotopy so that $f$ becomes compatible with the projection $S^{1} \times S^{2} \rightarrow S^{1}$, in other words, $f$ is a fiber homotopy equivalence of the trivial bundle $S^{1} \times S^{2} \rightarrow S^{1}$. It remains to show that it is fiber homotopy equivalent to an isomorphism of $S^{2}$-bundles with structure group $S O(3)$ over $S^{1}$. This boils down to showing that the obvious map $S O(3) \rightarrow S G(2)$ is 1connected. Analogously to the argument appearing in the proof of Theorem 8.2, 
but one dimension lower, one shows that it suffices to show that the (unstable) $J$-homomorphism $J^{\prime}: \pi_{1}(S O(2)) \rightarrow \pi_{3}\left(S^{2}\right)$ is bijective. By the Pontrjagin-Thom construction we obtain a bijection $\pi_{3}\left(S^{2}\right) \stackrel{\cong}{\rightarrow} \Omega_{1,3}^{\mathrm{fr}}$. Its composition with $J^{\prime}$ sends an element in $\pi_{1}(S O(2))$ to $S^{1} \subseteq \mathbb{R}^{3}$ with the induced framing and hence is surjective. Since $J^{\prime}$ is a surjective homomorphism of infinite cyclic groups, it is bijective.

(c) We have already shown in (a) that $S^{2} \times S^{2}$ is not strongly Borel. It is Borel by Theorem 0.11 (a).

(d) Suppose that $M$ is a Borel manifold. We have to check that conditions (di), (dii) and (diii) hold.

The $L$-class of $S^{k} \times S^{d}$ is concentrated in dimension 0 . We conclude from Theorem 0.21 that $S^{k} \times S^{d}$ can only be a Borel manifold if $H_{k+d-4 i}\left(S^{k} \times S^{d} ; \mathbb{Q}\right)$ is trivial for $i \in \mathbb{Z}, i \geq 1$. This implies that neither $k$ nor $d$ are divisible by four, i.e., condition (di) holds.

Suppose that $k$ is even. By (7.1) and Theorem 1.1 there exists an orientation preserving self-homotopy equivalence $f: S^{k} \times S^{d} \rightarrow S^{k} \times S^{d}$ which is transverse to $\{\bullet\} \times S^{d}$ such that the Arf invariant of the induced surgery problem $f^{-1}(\{\bullet\} \times$ $\left.S^{d}\right) \rightarrow\{\bullet\} \times S^{d}$ is non-trivial. We claim that there exists an orientation preserving homeomorphism $h: S^{k} \times S^{d} \rightarrow S^{k} \times S^{d}$ such that $f$ and $h$ induces the same isomorphism on $H_{n}\left(S^{k} \times S^{d}\right)$ for all $n \in \mathbb{Z}$. This is obvious by the Künneth formula in the case $k \neq d$ since there exists a homeomorphism $S^{n} \rightarrow S^{n}$ of degree -1 for all $n \geq 1$. In the case $k=d$, one has to take into account that $H_{k}(f)$ respects the intersection form on $H_{k}\left(S^{k} \times S^{k}\right)=H_{k}\left(S^{k}\right) \oplus H_{k}\left(S^{k}\right)=\mathbb{Z} \oplus \mathbb{Z}$ which is given by $\left(x_{1}, x_{2}\right) \cdot\left(y_{1}, y_{2}\right) \mapsto x_{1} y_{2}+x_{2} y_{1}$. This implies that $H_{k}(f)$ is given by one of the following matrices

$$
\left(\begin{array}{ll}
1 & 0 \\
0 & 1
\end{array}\right) \quad\left(\begin{array}{cc}
-1 & 0 \\
0 & -1
\end{array}\right) \quad\left(\begin{array}{cc}
0 & -1 \\
1 & 0
\end{array}\right) \quad\left(\begin{array}{cc}
0 & 1 \\
-1 & 0
\end{array}\right)
$$

Hence we can find the desired $h$. Now let $g_{k}: S^{k} \times S^{d} \rightarrow S^{k}$ be the composition $\operatorname{pr}_{S^{k}} \circ f \circ h^{-1}$ for the projection $\operatorname{pr}_{S^{k}}: S^{k} \times S^{d} \rightarrow S^{k}$. Obviously the Arf invariant of the codimension $k$ surgery problem obtained by making $g_{k}$ transversal to $\{\bullet\} \subseteq$ $S^{k}$ is one and $g_{k}$ restricted to $S^{k} \times\{\bullet\}$ defines an orientation preserving homotopy equivalence $S^{k} \times\{\bullet\} \rightarrow S^{k}$. The proof that (diii) holds is completely analogous. 
Now suppose that conditions (di), (dii) and (diii) are satisfied. We have to show that $S^{k} \times S^{d}$ is Borel what we will do by verifying the criterion appearing in Theorem 1.1. In view of assertion (a) and condition (di) we only have to deal with the case, where $k=2 \bmod 4$ or $d=2 \bmod 4$. We will only explain the most difficult case, where both $k=2 \bmod 4$ and $d=2 \bmod 4$ hold, the easier cases, where $k=2 \bmod 4$ and $d$ is odd or where $d=2 \bmod 4$ and $k$ is odd are then obvious.

Let $g_{k}: S^{k} \times S^{d} \rightarrow S^{k}$ be the map appearing in condition (dii). Define $f_{1}: S^{k} \times S^{d} \rightarrow S^{k} \times S^{d}$ to be $g_{k} \times \operatorname{pr}_{S^{d}}$ for $\operatorname{pr}_{d}: S^{k} \times S^{d} \rightarrow S^{d}$ the projection. This is an orientation preserving selfhomotopy equivalence satisfying $a_{1}\left(\left[f_{1}\right]\right)=0$ and $a_{2}\left(\left[f_{1}\right]\right)=1$. Using condition(diii) we construct an orientation preserving selfhomotopy equivalence $f_{2}: S^{k} \times S^{d} \rightarrow S^{k} \times S^{d}$ satisfying $a_{1}\left(\left[f_{2}\right]\right)=1$ and $a_{2}\left(\left[f_{2}\right]\right)=0$.

Obviously we can arrange that $f_{1}$ and $f_{2}$ induce the identity on $S^{k} \vee S^{d}$. This together with the identification

$$
\sigma_{k+d}: \mathcal{S}^{\text {top }}\left(S^{k} \times S^{d}\right) \cong \operatorname{ker}\left(H_{k+d}\left(S^{k} \times S^{d}, \mathbf{L}\langle 1\rangle\right) \rightarrow H_{k+d}(\{\bullet\}, \mathbf{L}\langle 1\rangle)\right)
$$

implies that the induced map on $\mathcal{S}^{\text {top }}\left(S^{k} \times S^{d}\right)$ the identity. Hence $f_{3}=f_{1} \circ f_{2}$ is the desired map because of the formula which has been communicated to us by Andrew Ranicki [29]

$$
\left[f_{2} \circ f_{1}\right]=\left(f_{2}\right)_{*}\left(\left[f_{1}\right]\right)+\left[f_{2}\right]=\left[f_{1}\right]+\left[f_{2}\right]
$$

and the fact that the isomorphism

$$
a_{1} \times a_{2}: \mathcal{S}^{\text {top }}\left(S^{k} \times S^{d}\right) \stackrel{\cong}{\longrightarrow} L_{k}(\mathbb{Z}) \oplus L_{d}(\mathbb{Z}),
$$

is compatible with the abelian group structures. This finishes the proof of Theorem 0.13 .

\section{On the Homotopy Type of Certain Low-Dimensional Manifolds}

We first compute the homology of the universal covering $\widetilde{M}$ for a manifold appearing in Problem 0.16. 
Lemma 8.1. Let $M$ and $K$ be manifolds as described in Problem 0.16. Then $n \geq 4$, if $k=1$ and $n \geq 5$, if $k=2$. Moreover

$$
H_{p}(\widetilde{M} ; \mathbb{Z}) \cong_{\mathbb{Z} \pi}\left\{\begin{array}{l}
\mathbb{Z} p=0, n-k ; \\
0 \quad 1 \leq p \leq 2 \\
0 \quad p \geq n-2, p \neq n-k,
\end{array}\right.
$$

where $\mathbb{Z}$ carries the trivial $\pi$-action. The $\mathbb{Z} \pi$-module $H_{3}(\widetilde{M})$ is finitely generated stably free if $n=6$.

Proof. Let $f: M \rightarrow B \pi$ be the classifying map for $\pi=\pi_{1}(M)$. In the sequel we identify $\pi=\pi_{1}(M)=\pi_{1}(K)$. The map is 3-connected because of $\pi_{2}(M)=0$. Let $\widetilde{f}: \widetilde{M} \rightarrow \widetilde{K}$ be the induced $\pi$-equivariant map on the universal coverings. The induced $\mathbb{Z} \pi$-chain map

$$
C_{*}(\widetilde{f}): C_{*}(\widetilde{M}) \rightarrow C_{*}(\widetilde{K})
$$

is homological 3-connected by the Hurewicz Theorem. This implies that its mapping cone is chain homotopy equivalent to a $\mathbb{Z} \pi$-chain complex whose chain modules are trivial in dimensions $\leq 3$. Therefore we obtain isomorphisms

$$
H_{p}(f ; \mathbb{Z}): H_{p}(M ; \mathbb{Z}) \stackrel{\cong}{\rightrightarrows} H_{p}(B \pi ; \mathbb{Z})
$$

and

$$
H_{p}(\widetilde{f} ; \mathbb{Z}): H_{p}(\widetilde{M} ; \mathbb{Z}) \stackrel{\cong}{\rightrightarrows} H_{p}(E \pi ; \mathbb{Z})
$$

for $p \leq 2$ and the induced $\mathbb{Z} \pi$-chain map

$$
C^{n-*}(\widetilde{f}): C^{n-*}(\widetilde{K}) \rightarrow C^{n-*}(\widetilde{M})
$$

induces isomorphism for $n-p \leq 2$

$$
H_{p}\left(C^{n-*}(\widetilde{f})\right): H_{p}\left(C^{n-*}(\widetilde{K})\right) \stackrel{\cong}{\rightrightarrows} H_{p}\left(C^{n-*}(\widetilde{M})\right) .
$$

Obviously $H_{p}(\widetilde{K} ; \mathbb{Z})=H_{p}\left(C_{*}(\widetilde{K})\right)$ is $\mathbb{Z} \pi$-isomorphic to the trivial $\mathbb{Z} \pi$-module for $p=0$ and is trivial for $p \neq 0$. Recall that we have the Poincaré $\mathbb{Z} \pi$-chain homotopy equivalences

$$
\begin{aligned}
- & \cap[M]: C^{n-*}(\widetilde{M}) \rightarrow C_{*}(\widetilde{M}) ; \\
-\cap[K]: C^{k-*}(\widetilde{K}) & \rightarrow C_{*}(\widetilde{K}) .
\end{aligned}
$$

By Poincaré duality applied to $K$ we conclude that $H_{p}\left(C^{n-*}(\widetilde{K})\right)$ is $\mathbb{Z} \pi$-isomorphic to $\mathbb{Z}$ for $n-p=k$ and is trivial for $n-p \neq k$. Hence $H_{p}\left(C^{n-*}(\widetilde{M})\right)$ is $\mathbb{Z} \pi$-isomorphic to $\mathbb{Z}$ for $n-p=k$ and is trivial for $n-p \in\{0,1,2\}, n-p \neq k$. 
From Poincaré duality applied to $\widetilde{M}$ we conclude that $H_{p}(\widetilde{M} ; \mathbb{Z})$ is $\mathbb{Z} \pi$-isomorphic to $\mathbb{Z}$ for $p=n-k$ and is trivial for $p \in\{n, n-1, n-2\}, p \neq n-k$. We already know that $H_{p}(\widetilde{M} ; \mathbb{Z})=H_{p}(\widetilde{K} ; \mathbb{Z})=0$ for $p=1,2$. This implies $n-k \notin\{1,2\}$ since $\mathbb{Z}$ is not trivial. Hence $n \geq 4$ if $k=1$ and $n \geq 5$ if $k=2$. It remains to show that the $\mathbb{Z} \pi$-module $H_{3}(\widetilde{M})$ is free if $n=6$. Let cone $\left(f_{*}\right)$ be the mapping cone of $C_{*}(\widetilde{f}): C_{*}(\widetilde{M}) \rightarrow C_{*}(\widetilde{K})$. The composition

$$
C_{*}(\widetilde{f}) \circ(-\cap[M]) \circ C^{6-*}(\widetilde{f}): C^{6-*}(\widetilde{K}) \rightarrow C_{*}(\widetilde{K})
$$

is obviously zero. Hence the $\mathbb{Z} \pi$-chain map

$$
(-\cap[M]) \circ C^{6-*}(\widetilde{f}): C^{6-*}(\widetilde{K}) \rightarrow C_{*}(\widetilde{M})
$$

induces in the obvious way a $\mathbb{Z} \pi$-chain map

$$
C^{6-*}(\widetilde{K}) \rightarrow \Sigma^{-1} \operatorname{cone}\left(f_{*}\right) .
$$

Let $D_{*}$ be its mapping cone. It inherits from the structure of a symmetric 6dimensional Poincaré chain complex on $C_{*}(\widetilde{M})$ the structure of a symmetric Poincaré chain complex on $D_{*}$ with Poincaré dimension 6 . This follows from [30, Proposition 4.1 on page 141]. The homology of $D_{*}$ is zero in dimensions different from 3 and $H_{3}\left(D_{*}\right)$ is $\mathbb{Z} \pi$-isomorphic to $H_{3}(\widetilde{M})$. Let $E_{*}$ be the $\mathbb{Z} \pi$ subchain complex of $D_{*}$ for which $E_{k}=0$ for $k \leq 2, E_{3}=\operatorname{ker}\left(d_{3}: D_{3} \rightarrow D_{2}\right)$ and $E_{k}=D_{k}$ for $k \geq 4$. Since

$$
0 \rightarrow \operatorname{ker}\left(d_{3}\right) \rightarrow D_{3} \stackrel{d_{3}}{\longrightarrow} D_{2} \stackrel{d_{2}}{\longrightarrow} D_{1} \stackrel{d_{1}}{\longrightarrow} D_{0} \rightarrow D_{-1} \rightarrow 0
$$

is exact, $E_{*}$ is a finitely generated free $\mathbb{Z} \pi$-chain complex. The inclusion $i: E_{*} \rightarrow$ $D_{*}$ is a homology equivalence and hence a $\mathbb{Z} \pi$-chain homotopy equivalence. In particular we can pullback the structure of a symmetric Poincaré chain complex of Poincaré dimension 6 on $D_{*}$ to $E_{*}$. Hence $E^{6-*}$ is a $\mathbb{Z} \pi$-chain complex which is concentrated in dimensions $-1,0,1,2$ and 3 , whose homology is zero in dimensions different from 3 and for which $H_{3}\left(E^{6-*}\right)$ is $\mathbb{Z} \pi$-isomorphic to $H_{3}(\widetilde{M})$. This implies that there is an exact sequence of $\mathbb{Z} \pi$-modules

$$
0 \rightarrow H_{3}(\widetilde{M}) \rightarrow E_{3}^{*} \rightarrow E_{4}^{*} \rightarrow E_{5}^{*} \rightarrow E_{6}^{*} \rightarrow E_{7}^{*} \rightarrow 0 .
$$

Hence $H_{3}(\widetilde{M})$ is a finitely generated stably free $\mathbb{Z} \pi$-module. This finishes the proof of Lemma 8.1.

Next we determine the homotopy type of $M$ in the case $n=5$. 
Theorem 8.2. Let $M$ and $K$ be as in Problem 0.16. Let $f: M \rightarrow K=B \pi$ be the classifying map for the $\pi$-covering $\widetilde{M} \rightarrow M$. Suppose $n \leq 5$. Then

(a) Let $k=2$. The homotopy type of $M$ is determined by its second StiefelWhitney class. Namely, there is precisely one fiber bundle $S^{3} \rightarrow E \stackrel{p}{\rightarrow}$ $K$ with structure group $S O(4)$ whose second Stiefel-Whitney class agrees under the isomorphism $H^{2}(f ; \mathbb{Z} / 2): H^{2}(K ; \mathbb{Z} / 2) \stackrel{\cong}{\rightrightarrows} H^{2}(M ; \mathbb{Z} / 2)$ with the second Stiefel-Whitney class of $M$. There exists a homotopy equivalence $g: M \rightarrow E$ such that $p \circ g$ and $f$ are homotopic;

(b) Suppose that $k=2$ and the second Stiefel-Whitney class of $M$ vanishes, or that $k=1$. Then there is a homotopy equivalence $g: M \rightarrow S^{n-k} \times B \pi$ such that $\mathrm{pr}_{B \pi} \circ g$ and $f$ are homotopic.

Proof. Obviously the second assertion is a special case of the first one. The first one is proven as follows.

We conclude from Lemma 8.1 that for $n \leq 5$ the universal covering $\widetilde{M}$ has the same homology as $S^{n-k}$ and that $\pi$ acts trivially on the homology of $\widetilde{M}$. Recall that $K$ is a model for $B \pi$. There is a fibration $F \rightarrow E \stackrel{p}{\rightarrow} K$ together with a homotopy equivalence $g: E \rightarrow M$ such that $f \circ g \simeq p$ and $F$ is homotopy equivalent to $\widetilde{M}$. Hence the fibration $p: E \rightarrow K$ has $S^{n-k}$ as fiber. Since the $\pi$-action on the homology of $\widetilde{M}$ is trivial, this spherical fibration is orientable, i.e., the fiber transport of this fibration $\pi=\pi_{1}(K) \rightarrow[F, F]$ is trivial.

Such fibrations over $K$ are classified by maps $u: K \rightarrow B S G(n-k)$, where $B S G(n-k)$ is the classifying space of the monoid $S G(n-k)$ of orientation preserving self-homotopy equivalences $u: S^{n-k} \rightarrow S^{n-k}$. We have $n-k \geq 3$. The space $S G(n-k)$ is connected and hence the space $B S G(n-k)$ is simply connected.

Suppose that $k=1$. Each map $S^{1} \rightarrow B S G(n-k)$ is trivial up to homotopy and the claim follows.

Now suppose $k=2$. Then $n \geq 5$ by Lemma 8.1 Hence $n=5$ because of the assumption $n \leq 5$. There is an obvious commutative diagram

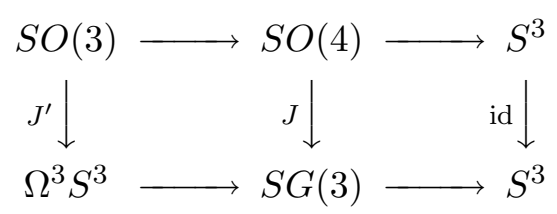


where the horizontal maps are fibrations and $J$ comes from the obvious action of $S O(4)$ on $S^{3}$. The following diagram commutes

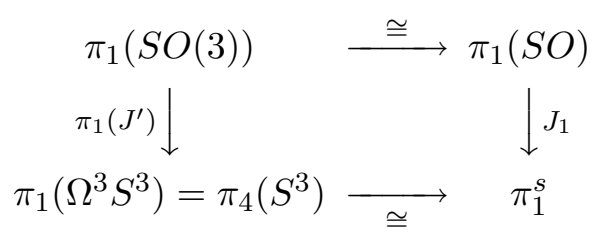

where the horizontal maps are given by stabilization and are isomorphisms and $J_{1}$ is the $J$-homomorphism. The abelian groups $\pi_{1}(S O)$ and $\pi_{1}^{s}$ are both isomorphic to $\mathbb{Z} / 2$. The map $J_{1}$ is bijective by [1]. Hence $J: S O(4) \rightarrow S G(3)$ is a map of connected space which induced an isomorphism on the fundamental groups. This implies that $B J: B S O(4) \rightarrow B S G(3)$ is a map of simply connected spaces inducing an isomorphism on $\pi_{2}$. Let $w_{2}: B S G(3) \rightarrow K(\mathbb{Z} / 2,2)$ be given by the second Stiefel-Whitney class. It and its composite $w_{2} \circ B J: B S O(4) \rightarrow K(\mathbb{Z} / 2,2)$ are 3 -connected. Since $K$ is 2-dimensional, we conclude that every orientable fibration $S^{3} \rightarrow E \rightarrow K$ is fiber homotopy equivalent to a fiber bundle $S^{3} \rightarrow E \rightarrow$ $K$ with structure group $S O(4)$ and two such fiber bundles with structure group $S O(4)$ over $K$ are isomorphic if and only if their second Stiefel-Whitney classes agree.

We have $H^{2}(f ; \mathbb{Z} / 2)\left(w_{2}(p)\right)=w_{2}(M)$ since for a vector bundle $\xi: E^{\prime} \rightarrow K$ we get a decomposition $T E^{\prime} \mid K \cong \xi \oplus T K$ and the Stiefel-Whitney classes of $K$ are trivial.

This finishes the proof of Theorem 8.2.

In dimension $n=6$ we can at least compute the homology of the universal covering up to stable isomorphism.

Lemma 8.3. Let $M$ and $K$ be as in Problem 0.16. Suppose $n=6$. Let $\chi(M)$ be the Euler characteristic.

$$
\begin{aligned}
& \text { Then }-\chi(M)+\left(1+(-1)^{k}\right) \cdot \chi(K) \geq 0 \text { and we get } \\
& \qquad H_{p}(\widetilde{M} ; \mathbb{Z}) \cong \mathbb{Z}_{\pi} \begin{cases}\mathbb{Z} p=0, n-k ; \\
0 & 0 \leq p \leq 2 ; \\
0 & p \geq n-2, p \neq n-k .\end{cases}
\end{aligned}
$$


and for some $s \geq 0$

$$
H_{3}(\widetilde{M} ; \mathbb{Z}) \oplus \mathbb{Z} \pi^{s} \cong_{\mathbb{Z} \pi} \mathbb{Z} \pi^{-\chi(M)+\left(1+(-1)^{k}\right) \cdot \chi(K)} \oplus \mathbb{Z} \pi^{s} .
$$

Proof. Because of Lemma 8.1 it suffices to show for the finitely generated stably free $\mathbb{Z} \pi$-module $H_{3}(\widetilde{M})$ that the classes of $H_{3}(\widetilde{M})$ and $\mathbb{Z} \pi^{-\chi(M)+\left(1+(-1)^{k}\right) \cdot \chi(K)}$ agrees in $K_{0}(\mathbb{Z} \pi)$.

We obtain a finite projective resolution for the trivial $\mathbb{Z} \pi$-module $\mathbb{Z}$ by $C_{*}(\widetilde{K})$ and get in $\widetilde{K}_{0}(\mathbb{Z} \pi)$

$$
[\mathbb{Z}]=\chi(K) \cdot[\mathbb{Z} \pi] .
$$

Therefore every homology module $H_{p}(\widetilde{M})$ for $p \neq 3$ possesses a finite projective $\mathbb{Z} \pi$-resolution and hence defines an element in $K_{0}(\mathbb{Z} \pi)$. This automatically implies that the same is true for $H_{3}(\widetilde{M})$. We compute in $K_{0}(\mathbb{Z} \pi)$

$$
\begin{aligned}
{\left[H_{3}(\widetilde{M})\right] } & =-\sum_{k=0}^{6}(-1)^{k} \cdot\left[H_{k}(\widetilde{M})\right]+[\mathbb{Z}]+(-1)^{6-k}[\mathbb{Z}] \\
& =-\sum_{k=0}^{6}(-1)^{k} \cdot\left[C_{k}(\widetilde{M})\right]+\left(1+(-1)^{k}\right) \cdot[\mathbb{Z}] \\
& =-\chi(M) \cdot[\mathbb{Z} \pi]+\left(1+(-1)^{k}\right) \cdot \chi(K) \cdot[\mathbb{Z} \pi] \\
& =\left(-\chi(M)+\left(1+(-1)^{k}\right) \cdot \chi(K)\right) \cdot[\mathbb{Z} \pi] .
\end{aligned}
$$

Now Lemma 8.3 follows.

\section{On the Classification of Certain Low-Dimensional Manifolds}

Theorem 9.1. Let $M$ and $K$ be as in Problem 0.16. Suppose $k=2$.

Then $n \geq 5$ and there is an isomorphism of abelian groups

$$
\mathcal{S}^{\text {top }}(M) \stackrel{\cong}{\rightrightarrows} \mathbb{Z} / 2 .
$$

If $f: M \rightarrow M$ is an orientation preserving self-homotopy equivalence and $g: N \rightarrow$ $M$ is an orientation preserving homotopy equivalence of manifolds, then we obtain in $\mathcal{S}^{\text {top }}(M)$

$$
[g \circ f]=[g]+[f] .
$$

Proof. Notice for the sequel that the Farrell-Jones Conjecture for algebraic $K$ and $L$-theory holds for $\pi_{1}(K)$ (see [14]). 
The Atiyah-Hirzebruch spectral sequence yields two commutative diagrams with exact columns respectively rows

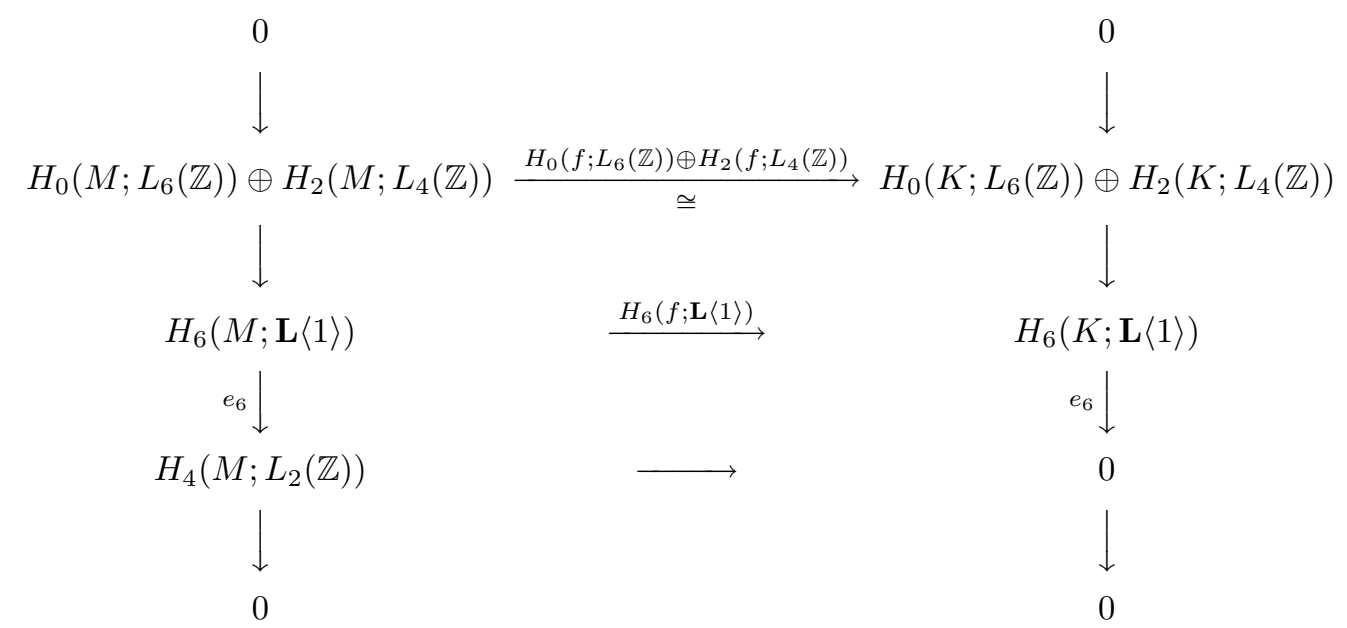

and

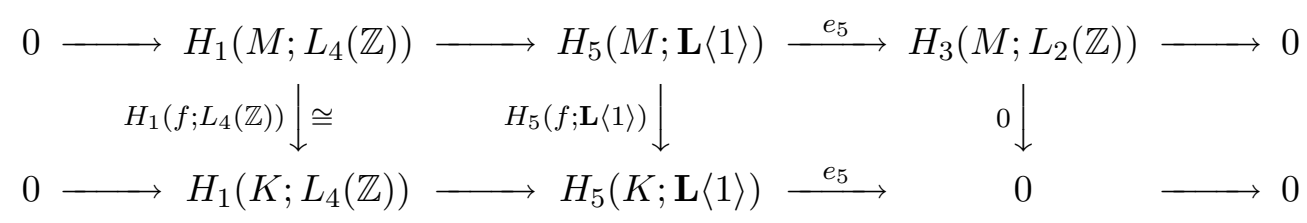

We conclude that the restriction of

$$
e_{n}: H_{n}(M ; \mathbf{L}\langle 1\rangle) \rightarrow H_{n-2}\left(M ; L_{2}(\mathbb{Z})\right)
$$

to the kernel of

$$
\left.H_{n}(f ; \mathbf{L}\langle 1\rangle)\right): H_{n}(M ; \mathbf{L}\langle 1\rangle) \rightarrow H_{n}(K ; \mathbf{L}\langle 1\rangle)
$$

is bijective. We conclude from Theorem 1.2 that the following composition is an isomorphism for $n=\operatorname{dim}(M)$.

$$
\mathcal{S}^{\text {top }}(M) \stackrel{\sigma_{n}}{\longrightarrow} H_{n}(M ; \mathbf{L}\langle 1\rangle) \stackrel{e_{n}}{\longrightarrow} H_{n-2}\left(M ; L_{2}(\mathbb{Z})\right) .
$$

We have the following composition of isomorphisms

$$
H^{2}\left(K ; L_{2}(\mathbb{Z})\right) \stackrel{H^{2}\left(f ; L_{2}(\mathbb{Z})\right)}{\longrightarrow} H^{2}\left(M ; L_{2}(\mathbb{Z})\right) \stackrel{-\cap[M]}{\longrightarrow} H_{n-2}\left(M ; L_{2}(\mathbb{Z})\right) .
$$

Since $k=2$ by assumption, we get

$$
H^{2}\left(K ; L_{2}(\mathbb{Z})\right) \cong L_{2}(\mathbb{Z}) \cong \mathbb{Z} / 2 .
$$

If $f: M \rightarrow M$ is an orientation preserving self-homotopy equivalence and $g: N \rightarrow M$ orientation preserving homotopy equivalence of manifolds, then we 
obtain in $\mathcal{S}^{\text {top }}(M)$ the formlua which has been communicated to us by Andrew Ranicki [29]

$$
[f \circ g]=f_{*}[g]+[f]
$$

Since any automorphism of the group $\mathbb{Z} / 2$ is the identity, this finishes the proof of Theorem 9.1.

Now we are ready to prove Theorem 0.18 and Theorem 0.19 (a).

Proof. In view of Theorem 8.2, Theorem 9.1 and Theorem 1.1 it remains to show that there exists a homotopy equivalence $f: M \rightarrow M$ inducing the up to conjugation the identity on the fundamental group which does not represent the trivial element id: $M \rightarrow M$ in $\mathcal{S}^{\text {top }}(M)$. We want to conclude this from Theorem 2.2 (a) and (b) As soon as we have provided the necessary data, the claim follows from Theorem 2.2 (a) since the 2-dimensional torus with an appropriate framing yields an element $\Omega_{2,2+d}^{\mathrm{fr}}$ for $d \geq 1$ whose image under the Arf invariant $\operatorname{map} \Omega_{2,2+d}^{\mathrm{fr}} \rightarrow \mathbb{Z} / 2$ is non-trivial.

Since the dimension of $K$ is less or equal to the connectivity of $S^{d}$, we can choose a map $i: K \rightarrow S^{d}$ such that $p \circ i$ is homotopic to the identity. By the homotopy lifting property we can arrange that $p \circ i=\mathrm{id}_{K}$. In particular we see that $i: K \rightarrow M$ is an embedding. It remains to construct the map $\alpha: S^{d} \rightarrow M$.

Let $\nu=\nu(i)$ be the normal bundle of $i: K \rightarrow M$ and $D \nu$ be the associated disk bundle. Let $j_{1}: D^{d} \subseteq D \nu \subseteq M$ be the inclusion of the fiber of a point $x \in K$. Obviously $D^{d}$ intersects $K$ transversally and the intersection consists of one point. It suffices to show that the map $j_{0}: S^{d-1}=\partial D^{d} \rightarrow M-K$ given by restricting $j_{1}$ to $S^{d-1}$ is nullhomotopic because then we can extend $j_{0}$ to a map $j_{2}: D^{d} \rightarrow M-K$ and can define the desired map $\phi$ by $j_{2} \cup_{j_{0}} j_{1}: S^{d}=D^{d} \cup_{S^{d-1}} D^{d} \rightarrow M$.

The map $i: K \rightarrow M$ and the map $p: M \rightarrow K$ induce isomorphisms on the fundamental groups. Fix a universal covering $\widetilde{K} \rightarrow K$. Its pullback with $p: M \rightarrow$ $K$ is the universal covering $\widetilde{M} \rightarrow M$. Obviously we get $\pi$-equivariant maps $\widetilde{i}: \widetilde{K} \rightarrow \widetilde{M}$ and $\widetilde{p}: \widetilde{M} \rightarrow \widetilde{K}$ covering $i$ and $p$ and satisfying $\widetilde{p} \circ \widetilde{i}=\mathrm{id}_{\widetilde{K}}$. Let $\widetilde{j_{1}}: D^{d} \rightarrow \widetilde{M}$ and $\widetilde{j_{0}}: S^{d-1} \rightarrow \widetilde{M}$ be lifts of $j_{1}$ and $j_{0}$ with $\left.\widetilde{j_{1}}\right|_{S^{d-1}}=\widetilde{j_{0}}$. The map $\pi_{d-1}(\widetilde{M}-\widetilde{i}(\widetilde{K})) \rightarrow \pi_{d-1}(M-i(K))$ is induced by a covering map and hence an isomorphism because of $d-1 \geq 2$. It sends the class of $\widetilde{j_{0}}$ to the class of $j_{0}$. Hence it suffices to show that the class of $\widetilde{j_{0}}$ in $\pi_{d-1}(\widetilde{M}-\widetilde{i}(\widetilde{K}))$ is zero. Its image under the map $\pi_{d-1}(\widetilde{M}-\widetilde{i}(\widetilde{K})) \rightarrow \pi_{d-1}\left(\pi_{d-1}(\widetilde{M})\right)$ is zero, a nullhomotopy for 
the image is given by $\widetilde{j_{1}}$. Since $\widetilde{M}$ is simply connected and its homology is trivial in dimensions $\leq d-2$ by Lemma 8.1 , the space $\widetilde{M}$ is $(d-2)$-connected. Since the codimension of $\widetilde{i}(\widetilde{K})$ in $\widetilde{M}$ is $d$, the inclusion $\widetilde{M}-\widetilde{i}(\widetilde{K}) \rightarrow \widetilde{M}$ is $(d-1)$-connected and hence $\widetilde{M}-\widetilde{i}(\widetilde{K})$ is $(d-2)$-connected. Therefore the Hurewicz homomorphism

$$
\pi_{d-1}(\widetilde{M}-\widetilde{i}(\widetilde{K})) \stackrel{\cong}{\rightrightarrows} H_{d-1}(\widetilde{M}-\widetilde{i}(\widetilde{K}))
$$

is an isomorphism. The image of the class of $\widetilde{j_{0}}$ under the Hurewicz homomorphism is send to zero under the map $H_{d-1}(\widetilde{M}-\widetilde{i}(\widetilde{K})) \rightarrow H_{d-1}(\widetilde{M})$. Hence it remains to show that this map is injective. By the long exact sequence of the pair it suffices to prove that $H_{d}(\widetilde{M}) \rightarrow H_{d}(\widetilde{M}, \widetilde{M}-\widetilde{i}(\widetilde{K}))$ is surjective. By Poincaré duality we get a commutative diagram with isomorphisms as vertical maps

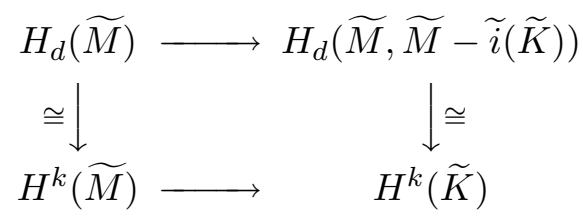

where the lower horizontal arrow is induced by $\widetilde{i}$ and is split surjective because of $\widetilde{p} \circ \widetilde{i}=\mathrm{id}_{\widetilde{K}}$. This finishes the proofs of Theorem 0.18 and Theorem 0.19 (a).

Next we give the proof of Theorem 0.19 (b).

Proof. Let $M$ be a closed topological oriented 6-manifold with $\pi_{1}(M) \cong \pi_{1}(K)$, where $K$ is a $K(\pi, 1)$-manifold of dimension $\leq 2$, and $\pi_{2}(M)=0$. We want to prove that the $\mathbb{Z} \pi$-isomorphism class of the intersection form on $H_{3}(\widetilde{M})$ determines the homeomorphism type. The normal 2-type of $M$ in the sense of Kreck [22] is

$$
K \times \text { BTopSpin } \stackrel{\mathrm{pr}_{2}}{\longrightarrow} \text { BTopSpin } \stackrel{P}{\longrightarrow} \text { BSTOP }
$$

if $M$ is a topological Spin-manifold and

$$
K \times \text { BTopSpin } \stackrel{E \times P}{\longrightarrow} B S T O P \times B S T O P \stackrel{\oplus}{\longrightarrow} B S T O P
$$

if $M$ is not a topological Spin-manifold, where $E$ is a vector bundle over $K$ with $w_{2}(E) \neq 0$. This normal 2-type is determined by $\pi=\pi_{1}(K)$ and $w_{2}=w_{2}(M) \epsilon$ $\mathbb{Z} / 2$ and so we denote it by $B\left(\pi, w_{2}\right)$. Notice that $(M ; \bar{\nu})$ determines a class in the bordism group $\Omega_{6}\left(B\left(\pi, w_{2}\right)\right)$.

Now we want to apply [23, Corollary 3]. It says that if $\left(M^{\prime} ; \bar{\nu}\right)$ is another normal 2-smoothing in $B\left(\pi, w_{2}\right)$, then $M$ and $M^{\prime}$ are homeomorphic, where the 
homeomorphism is compatible with the maps on $\pi_{1}$, if and only if the pairs determine the same class in $\Omega_{6}\left(B\left(\pi, w_{2}\right)\right)$ and the intersection form together with quadratic refinement on $K\left(\pi_{3}(M)\right) \rightarrow \pi_{3}\left(B\left(\pi, w_{2}\right)\right)=\pi_{3}(M)$, which by Lemma 8.1 is stably free and by Poincaré duality unimodular, are isomorphic.

Since $\left\langle w_{4}\left(B\left(\pi, w_{2}\right)\right), \pi_{4}\left(B\left(\pi, w_{2}\right)\right)\right\rangle \neq 0$ we have to consider the quadratic refinement with values in $\mathbb{Z} \pi /\langle x+\bar{x}, 1\rangle$. This is a quadratic refinement with respect to a form parameter in the sense of Bak [2]. Since $\pi$ has no element of order 2 and the quadratic form on $\pi_{3}(M)$ takes values in $\mathbb{Z}[\pi] /\langle a+\bar{a}, 1\rangle=0$, the quadratic form is determined by the intersection form. So it remains to show that the intersection form determines the bordism class in $\Omega_{6}\left(B\left(\pi, w_{2}\right)\right)$.

The Atiyah-Hirzebruch spectral sequence shows that $\Omega_{6}\left(B\left(\pi, w_{2}\right)\right) \cong H_{2}(K ; \mathbb{Z})$ ). If $\operatorname{dim}(K)=1$, then $\Omega_{6}\left(B\left(\pi, w_{2}\right)\right)$ is trivial and the claim follows.

Suppose $\operatorname{dim}(K)=2$. Then $\Omega_{6}\left(B\left(\pi, w_{2}\right)\right)$ is isomorphic to $\mathbb{Z}$. Consider the following composite

$$
\Omega_{6}\left(B\left(\pi, w_{2}\right)\right) \stackrel{\alpha}{\rightarrow} \Omega_{6}(B \pi) \stackrel{\sigma}{\rightarrow} L^{6}(\mathbb{Z} \pi)
$$

where $\alpha$ is induced by the obvious map $B\left(\pi, w_{2}\right) \rightarrow B \pi$ and the second map is given by the symmetric signature in the sense of Ranicki [31], [32]. The argument in the proof of Lemma 8.1 shows that the class $\beta \circ \alpha([M ; \bar{\nu}]$ which is given by the chain complex of the associated $\pi$-covering of $M$ and the Poincaré chain homotopy equivalence is the same as the class represented by the intersection form, since the two are obtained from one another by algebraic surgery. Hence the intersection from determines $\beta \circ \alpha\left([M ; \bar{\nu}]\right.$. Since $\Omega_{6}\left(B\left(\pi, w_{2}\right)\right)$ is torsionfree, it remains to show that $\beta \circ \alpha$ is rationally injective.

There is the following commutative diagram of $\mathbb{Z}$-graded rational vector spaces as explained in [25, page 728$]$

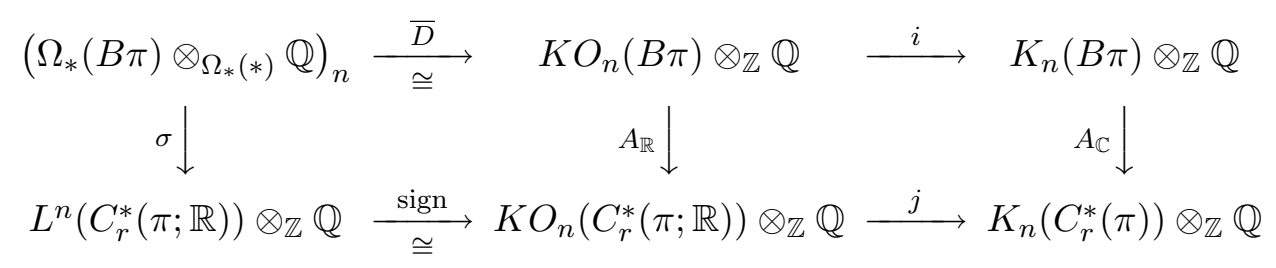

The map $A_{\mathbb{C}}$ is the assembly map appearing in the Baum Connes Conjecture and is known to be an isomorphism. The map $i$ is a change of rings map and known 
to be rationally injective. The map $\sigma$ factorizes as

$$
\left(\Omega_{*}(B \pi) \otimes_{\Omega_{*}(*)} \mathbb{Q}\right)_{n} \rightarrow L^{n}(\mathbb{Z} \pi) \otimes_{\mathbb{Z}} \mathbb{Q} \rightarrow L^{n}\left(C_{r}^{*}(\pi ; \mathbb{R})\right) \otimes_{\mathbb{Z}} \mathbb{Q} .
$$

Hence it suffices to show that the composite

$$
\Omega_{6}\left(B\left(\pi, w_{2}\right)\right) \otimes_{\mathbb{Z}} \mathbb{Q} \stackrel{\alpha \otimes_{\mathbb{Z}} \mathrm{id}_{\mathbb{Q}}}{\longrightarrow} \Omega_{6}(B \pi) \otimes_{\mathbb{Z}} \mathbb{Q} \rightarrow \Omega_{*}(B \pi) \otimes_{\Omega_{*}(*)} \mathbb{Q} \rightarrow K O_{6}(B \pi) \otimes_{\mathbb{Z}} \mathbb{Q}
$$

is injective. This follows from a spectral sequence argument. This shows that the intersection form determines the homeomorphism type.

The next question is which unimodular forms $\lambda$ on stably free $\mathbb{Z}\left[\pi_{1}\right]$-modules $V$ can be realized as intersection forms of manifolds under consideration. Since, if $(V, \lambda)$ can be realized, and $(V, \lambda)$ splits off a hyperbolic form, i.e., $(V, \lambda)=$ $\left(V^{\prime}, \lambda^{\prime}\right) \perp H$, then $\left(V^{\prime}, \lambda^{\prime}\right)$ can be realized by surgery on the hyperbolic plane, the realization problem is reduced to the stable realization problem: Which elements in $\widetilde{L}_{6}\left(\pi_{1}(K)\right)$ can be realized by a stable homeomorphism class?

If $w_{2}=0$, the answer is: All. The reason is that we have a commutative diagram

$$
\begin{gathered}
\Omega_{4}^{\text {TopSpin }} \stackrel{\cong}{\cong} L_{4}(\{e\}) \\
\cong \downarrow \times K \quad \downarrow \cong \\
\Omega_{6}^{\text {TopSpin }}(K) \longrightarrow \widetilde{L}_{6}\left(\pi_{1}(K)\right)
\end{gathered}
$$

If $w_{2} \neq 0$ we don't know the answer.

\section{A Necessary Condition for Being a Borel Manifold}

Next we prove Theorem 0.21 .

Proof. For every homology theory satisfying the disjoint union axiom and hence in particular for $H_{*}(-; \mathbf{L}\langle 1\rangle)$ there is a natural Chern character (see Dold [8]).

$$
\operatorname{ch}_{n}(X): \bigoplus_{i \in \mathbb{Z}, i \geq 1} H_{n-4 i}(X ; \mathbb{Q}) \stackrel{\cong}{\rightrightarrows} H_{n}(X ; \mathbf{L}\langle 1\rangle) \otimes_{\mathbb{Z}} \mathbb{Q}
$$

For an $n$-dimensional manifold $M$ the composite

$$
\mathcal{S}^{\mathrm{top}}(M) \stackrel{\sigma_{n}}{\longrightarrow} H_{n}(M ; \mathbf{L}\langle 1\rangle) \rightarrow H_{n}(M ; \mathbf{L}\langle 1\rangle) \otimes_{\mathbb{Z}} \mathbb{Q} \stackrel{\operatorname{ch}_{n}(M)^{-1}}{\longrightarrow} \bigoplus_{i \in \mathbb{Z}, i \geq 1} H_{n-4 i}(M ; \mathbb{Q})
$$


sends the class of an orientation preserving homotopy equivalence $f: N \rightarrow M$ to the element $\left\{f_{*}(\mathcal{L}(N) \cap[N])-\mathcal{L}(M) \cap[M] \mid i \in \mathbb{Z}, i \geq 1\right\}$. (see [33, Example 18.4 on page 198]).

Now suppose that $M$ is a Borel manifold. Then by Theorem 1.1 the operation of the group of homotopy classes of self-homotopy equivalence $M \rightarrow M$ which induce the identity on the fundamental group up to conjugation on the topological structure set $\mathcal{S}^{\text {top }}(M)$ is transitive. This implies that this group acts also transitive on the image of the composite

$$
\mathcal{S}^{\text {top }}(M) \stackrel{\sigma_{n}}{\longrightarrow} H_{n}(M ; \mathbf{L}\langle 1\rangle) \rightarrow H_{n}(M ; \mathbf{L}\langle 1\rangle) \otimes_{\mathbb{Z}} \mathbb{Q} \stackrel{\operatorname{ch}_{n}(M)^{-1}}{\longrightarrow} \bigoplus_{i \in \mathbb{Z}, i \geq 1} H_{n-4 i}(M ; \mathbb{Q}) .
$$

This image is obviously an abelian subgroup of $\bigoplus_{i \in \mathbb{Z}, i \geq 1} H_{n-4 i}(N ; \mathbb{Q})$ and agrees with the set

$$
S:=\left\{f_{*}(\mathcal{L}(M) \cap[M])-\mathcal{L}(M) \cap[M] \mid[f] \in \operatorname{ho-aut}_{\pi}(M)\right\} .
$$

By the exactness of the surgery sequence the $\mathbb{Q}$-submodule generated by $S$ contains the kernel of the map induced by the classifying map $c: M \rightarrow B \pi$

$$
c_{*}: \bigoplus_{i \in \mathbb{Z}, i \geq 1} H_{n-4 i}(M ; \mathbb{Q}) \rightarrow \bigoplus_{i \in \mathbb{Z}, i \geq 1} H_{n-4 i}(B \pi ; \mathbb{Q}) .
$$

Now we are ready to prove Theorem 0.20 .

Proof. We first show that the set of homotopy classes of orientation preserving homotopy equivalences $f: E \rightarrow E$ which induce up to conjugation the identity on the fundamental group is finite. Since $K$ is aspherical and $p: E \rightarrow K$ induces an isomorphism on the fundamental groups because of $d \geq 2$, the maps $p$ and $p \circ f$ are homotopic. By the homotopy lifting property we can assume that $p \circ f=p$ holds. Hence it suffices to show that the set of fiber homotopy classes of fiber homotopy equivalences $f: E \rightarrow E$ which cover the identity $\operatorname{id}_{K}: K \rightarrow K$ and induce a map of degree one on the fibers is finite. By elementary obstruction theory this follows if the $i$-th homotopy group of the space $S G\left(S^{d}\right)$ of self-maps $S^{d} \rightarrow S^{d}$ of degree one is finite for $i \leq k$. There is an obvious fibration $\Omega^{d} S^{d} \rightarrow$ $S G\left(S^{d}\right) \rightarrow S^{d}$. The long exact homotopy sequence yields the exact sequence $\pi_{i+d}\left(S^{d}\right) \rightarrow \pi_{i}\left(S G\left(S^{d}\right)\right) \rightarrow \pi_{i}\left(S^{d}\right)$, where we take the obvious base points. If $d$ is odd, $\pi_{j}\left(S^{d}\right)$ is finite for all $j \geq 0$, and, if $d$ is even, $\pi_{j}\left(S^{d}\right)$ is finite for $j \leq 2 d-2$. 
This has been proved by Serre [34]. Hence $\pi_{i}\left(S G\left(S^{d}\right)\right)$ is finite if $i \geq 0$ and $d$ is odd or if $i \leq d-2$.

Since $K$ is aspherical and there is a map $i: K \rightarrow E$ with $p \circ i \simeq \mathrm{id}_{K}$, the kernel of the map $c_{*}: H_{k+d-4 i}(E) \rightarrow H_{k+d-4 i}\left(B \pi_{1}(E) ; \mathbb{Q}\right)$ induced by the classifying map $c=p: E \rightarrow B \pi_{1}(E)=K$ contains $H_{k-4 i}(K ; \mathbb{Q})$. Now the claim follows from Theorem 0.21 because the abelian subgroup $S$ of a $\mathbb{Q}$-module appearing there is finite and hence trivial.

\section{Integral Homology Spheres}

In this section we prove Theorem 0.23

Proof. (a) Let $c: M \rightarrow S^{n}$ the collapse map which is a map of degree one. Since $M$ is by assumption a homology sphere, it induces an isomorphism on integral homology. By the Atiyah Hirzebruch spectral sequence it induces isomorphisms

$$
H_{p}(c ; \mathbf{L}\langle 1\rangle): H_{p}(M ; \mathbf{L}\langle 1\rangle) \rightarrow H_{p}\left(S^{n} ; \mathbf{L}\langle 1\rangle\right)
$$

for all $p \in \mathbb{Z}$. We obtain the following commutative diagram whose vertical arrows are parts of the long exact surgery sequence (1.3) where we here use the decoration $s$, i.e., we take the Whitehead torsion into account.

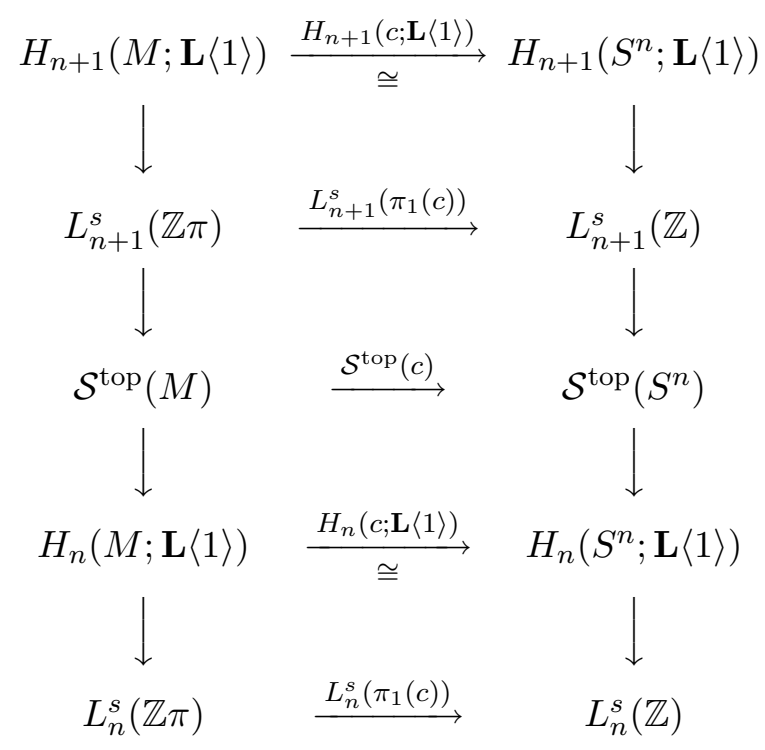


By the Poincaré Conjecture $\mathcal{S}^{\text {top }}\left(S^{n}\right)$ is trivial. An easy diagram chase together with Theorem 1.1 shows that $M$ is strongly Borel if and only if

$$
H_{n+1}(c ; \mathbf{L}\langle 1\rangle): H_{n+1}(M ; \mathbf{L}\langle 1\rangle) \rightarrow L_{n+1}^{s}(\mathbb{Z} \pi)
$$

is surjective. Notice that the natural map $\mathbf{L}^{s} \rightarrow \mathbf{L}$ is a homotopy equivalence since the Whitehead group of the trivial group is zero. The following diagram

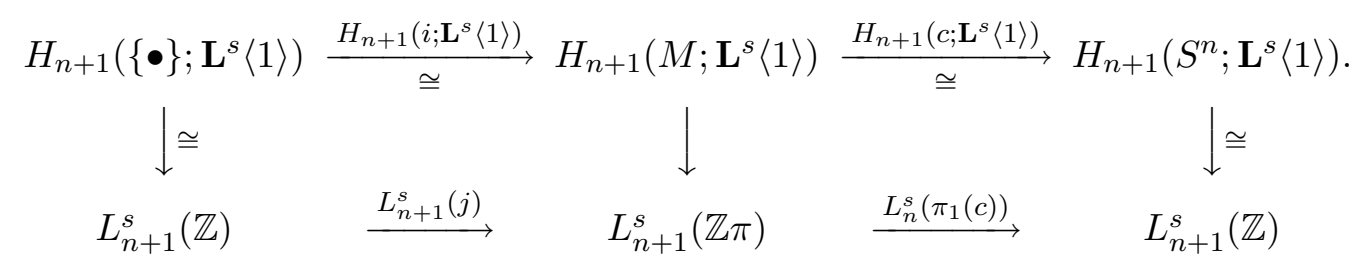

commutes, where $i:\{\bullet\} \rightarrow M$ and $j: \mathbb{Z} \rightarrow \mathbb{Z} \pi$ denote the obvious inclusions and all maps marked with $\cong$ are isomorphisms by the Atiyah-Hirzebruch spectral sequence. Obviously $L_{n}^{s}\left(\pi_{1}(c)\right) \circ L_{n+1}^{s}(j)$ is the identity. Hence $L_{n+1}^{s}(j)$ is bijective if and only if $H_{n+1}(c ; \mathbf{L}\langle 1\rangle)$ is surjective. This shows that $M$ is strongly Borel if and only if $L_{n+1}^{s}(j)$ is bijective.

(b) Suppose that $M$ is strongly Borel and a rational homology sphere. Since then $\mathcal{S}^{\text {top }}(M)=\{0\}$, the map $H_{n+1}\left(M ; \mathbf{L}^{s}\langle 1\rangle\right) \rightarrow L_{n+1}^{s}(\mathbb{Z} \pi)$ is surjective. Since this map factorizes as

$$
H_{n+1}\left(M ; \mathbf{L}^{s}\langle 1\rangle\right) \stackrel{H_{n+1}\left(f ; \mathbf{L}^{s}\langle 1\rangle\right)}{\longrightarrow} H_{n+1}\left(B \pi ; \mathbf{L}^{s}\langle 1\rangle\right) \rightarrow L_{n+1}^{s}(\mathbb{Z} \pi)
$$

for the classifying map $f: M \rightarrow B \pi$ and the latter map is rationally injective by assumption, the homomorphism $H_{n+1}\left(f ; \mathbf{L}^{s}\langle 1\rangle\right)$ is rationally surjective. Given a $C W$-complex $X$ we have the isomorphism given by the Chern character

$$
\bigoplus_{i \geq 1} H_{n+1-4 i}(M ; \mathbb{Q}) \stackrel{\cong}{\rightrightarrows} H_{n+1}\left(M ; \mathbf{L}^{s}\langle 1\rangle\right) \text {. }
$$

Hence the map $H_{n+1-4 i}(f ; \mathbb{Q}): H_{n+1-4 i}(M ; \mathbb{Q}) \rightarrow H_{n+1-4 i}(B \pi ; \mathbb{Q})$ is surjective for $i \geq 1$.

\section{REFERENCES}

[1] J. F. Adams. On the groups $J(X)$. IV. Topology, 5:21-71, 1966.

[2] A. Bak. K-theory of forms. Princeton University Press, Princeton, N.J., 1981.

[3] W. Browder. The Kervaire invariant of framed manifolds and its generalization. Ann. of Math. (2), 90:157-186, 1969.

[4] S. E. Cappell. A splitting theorem for manifolds. Invent. Math., 33(2):69-170, 1976. 
[5] S. Chang and S. Weinberger. On invariants of Hirzebruch and Cheeger-Gromov. Geom. Topol., 7:311-319 (electronic), 2003.

[6] M. M. Cohen. A course in simple-homotopy theory. Springer-Verlag, New York, 1973. Graduate Texts in Mathematics, Vol. 10.

[7] F. X. Connolly and J. F. Davis. The surgery obstruction groups of the infinite dihedral group. Geom. Topol., 8:1043-1078 (electronic), 2004.

[8] A. Dold. Relations between ordinary and extraordinary homology. Colloq. alg. topology, Aarhus 1962, 2-9, 1962.

[9] F. T. Farrell. Lectures on surgical methods in rigidity. Published for the Tata Institute of Fundamental Research, Bombay, 1996.

[10] F. T. Farrell. The Borel conjecture. In F. T. Farrell, L. Göttsche, and W. Lück, editors, High dimensional manifold theory, number 9 in ICTP Lecture Notes, pages 225-298. Abdus Salam International Centre for Theoretical Physics, Trieste, 2002. Proceedings of the summer school "High dimensional manifold theory" in Trieste May/June 2001, Number 1. http://www.ictp.trieste.it/ pub_off/lectures/vol9.html.

[11] F. T. Farrell and L. E. Jones. A topological analogue of Mostow's rigidity theorem. J. Amer. Math. Soc., 2(2):257-370, 1989.

[12] F. T. Farrell and L. E. Jones. Rigidity and other topological aspects of compact nonpositively curved manifolds. Bull. Amer. Math. Soc. (N.S.), 22(1):59-64, 1990.

[13] F. T. Farrell and L. E. Jones. Isomorphism conjectures in algebraic $K$-theory. J. Amer. Math. Soc., 6(2):249-297, 1993.

[14] F. T. Farrell and L. E. Jones. Topological rigidity for compact non-positively curved manifolds. In Differential geometry: Riemannian geometry (Los Angeles, CA, 1990), pages 229-274. Amer. Math. Soc., Providence, RI, 1993.

[15] F. T. Farrell and L. E. Jones. Rigidity for aspherical manifolds with $\pi_{1} \subset G L_{m}(\mathbb{R})$. Asian J. Math., 2(2):215-262, 1998.

[16] S. C. Ferry, A. A. Ranicki, and J. Rosenberg. A history and survey of the Novikov conjecture. In Novikov conjectures, index theorems and rigidity, Vol. 1 (Oberwolfach, 1993), pages 7-66. Cambridge Univ. Press, Cambridge, 1995.

[17] M. H. Freedman. The disk theorem for four-dimensional manifolds. In Proceedings of the International Congress of Mathematicians, Vol. 1, 2 (Warsaw, 1983), pages 647-663, Warsaw, 1984. PWN.

[18] M. H. Freedman and F. Quinn. Topology of 4-manifolds. Princeton University Press, Princeton, NJ, 1990.

[19] I. Hambleton and M. Kreck. Cancellation, elliptic surfaces and the topology of certain four-manifolds. J. Reine Angew. Math., 444:79-100, 1993.

[20] J. Hempel. 3-Manifolds. Princeton University Press, Princeton, N. J., 1976. Ann. of Math. Studies, No. 86.

[21] R. C. Kirby and L. C. Siebenmann. Foundational essays on topological manifolds, smoothings, and triangulations. Princeton University Press, Princeton, N.J., 1977. With notes by J. Milnor and M. F. Atiyah, Annals of Mathematics Studies, No. 88.

[22] M. Kreck. Surgery and duality. Ann. of Math. (2), 149(3):707-754, 1999. 
[23] M. Kreck. Cancellation for stable diffeomorphisms. preprint, 2004.

[24] M. Kreck and W. Lück. The Novikov Conjecture: Geometry and Algebra, volume 33 of Oberwolfach Seminars. Birkhäuser, 2005.

[25] E. Leichtnam, W. Lück, and M. Kreck. On the cut-and-paste property of higher signatures of a closed oriented manifold. Topology, 41(4):725-744, 2002.

[26] W. Lück. A basic introduction to surgery theory. In F. T. Farrell, L. Göttsche, and W. Lück, editors, High dimensional manifold theory, number 9 in ICTP Lecture Notes, pages 1-224. Abdus Salam International Centre for Theoretical Physics, Trieste, 2002. Proceedings of the summer school "High dimensional manifold theory" in Trieste May/June 2001, Number 1. http://www.ictp.trieste.it/ pub_off/lectures/vol9.html.

[27] W. Lück and H. Reich. The Baum-Connes and the Farrell-Jones Conjectures in K- and L-Theory. In Handbook of $K$-theory, volume 2, pages 703 - 842. Springer-Verlag, Berlin, 2005.

[28] J. Milnor. On simply connected 4-manifolds. In Symposium internacional de topología algebraica International symposi um on algebraic topology, pages 122-128. Universidad Nacional Autónoma de México and UNESCO, Mexico City, 1958.

[29] A. Ranicki. On the manifold structure set. in preparation, 2005.

[30] A. A. Ranicki. Algebraic L-theory. I. Foundations. Proc. London Math. Soc. (3), 27:101125,1973 .

[31] A. A. Ranicki. The algebraic theory of surgery. I. Foundations. Proc. London Math. Soc. (3), 40(1):87-192, 1980

[32] A. A. Ranicki. Exact sequences in the algebraic theory of surgery. Princeton University Press, Princeton, N.J., 1981.

[33] A. A. Ranicki. Algebraic L-theory and topological manifolds. Cambridge University Press, Cambridge, 1992.

[34] J.-P. Serre. Groupes d'homotopie et classes de groupes abéliens. Ann. of Math. (2), 58:258294, 1953.

[35] V. G. Turaev. Homeomorphisms of geometric three-dimensional manifolds. Mat. Zametki, 43(4):533-542, 575, 1988. translation in Math. Notes 43 (1988), no. 3-4, 307-312.

[36] F. Waldhausen. Algebraic K-theory of topological spaces. I. In Algebraic and geometric topology (Proc. Sympos. Pure Math., Stanford Univ., Stanford, Calif., 1976), Part 1, pages 35-60. Amer. Math. Soc., Providence, R.I., 1978.

[37] C. T. C. Wall. Classification of $(n-1)$-connected 2n-manifolds. Ann. of Math. (2), 75:163$189,1962$.

[38] C. T. C. Wall. Surgery on compact manifolds. American Mathematical Society, Providence, RI, second edition, 1999. Edited and with a foreword by A. A. Ranicki.

Matthias Kreck

Hausdorff Research Institute for Mathematics

University of Bonn

Poppelsdorfer Allee 45, 53115 Bonn, Germany 
E-mail: kreck@him.uni-bonn.de http://www.hausdorff-research-institute.uni-bonn.de/kreck Fax: 49 (0)228 734406

Wolfgang Lück

Fachbereich Mathematik, Universität Münster

Einsteinstr. 62, 48149 Münster, Germany

E-mail: lueck@math.uni-muenster.de

http://www.math.uni-muenster.de/u/lueck

Fax: 49 (0)251 8338370 